\title{
Schrödinger's Cat Paradox Resolution Using GRW Collapse Model: Von Neumann Measurement Postulate Revisited
}

\author{
Jaykov Foukzon', Alex Potapov², Elena Men'kova3 ${ }^{3}$, Stanislav Podosenov ${ }^{3}$ \\ ${ }^{1}$ Center for Mathematical Sciences, Israel Institute of Technology, Haifa, Israel \\ ${ }^{2}$ IRE RAS, Moscow, Russia \\ ${ }^{3}$ All-Russian Research Institute, Moscow, Russia \\ Email: jaykovfoukzon@list.ru, potapov@cplire.ru, e_menkova@mail.ru, podosenov@mail.ru
}

How to cite this paper: Foukzon, J., Potapov, A., Men'kova, E. and Podosenov, S. (2017) Schrödinger's Cat Paradox Resolution Using GRW Collapse Model: Von Neumann Measurement Postulate Revisited. Journal of Applied Mathematics and Physics, 5, 494-521.

https://doi.org/10.4236/jamp.2017.52044

Received: November 1, 2016

Accepted: February 21, 2017

Published: February 24, 2017

Copyright $\odot 2017$ by authors and Scientific Research Publishing Inc. This work is licensed under the Creative Commons Attribution International License (CC BY 4.0).

http://creativecommons.org/licenses/by/4.0/

\begin{abstract}
In his famous thought experiment, Schrôdinger (1935) imagined a cat that measures the value of a quantum mechanical observable with its life. Since Schrödinger's time, no any interpretations or modifications of quantum mechanics have been proposed which give clear unambiguous answers to the questions posed by Schrödinger's cat of how long superpositions last and when (or whether) they collapse? In this paper appropriate modification of quantum mechanics is proposed. We claim that canonical interpretation of the wave function $\psi=c_{1} \psi_{1}+c_{2} \psi_{2}$ is correct only when the supports of the wave functions $\psi_{1}$ and $\psi_{2}$ essentially overlap. When the wave functions $\psi_{1}$ and $\psi_{2}$ have separated supports (as in the case of the experiment that we are considering in this paper) we claim that canonical interpretation of the wave function $\psi=c_{1} \psi_{1}+c_{2} \psi_{2}$ is no longer valid for a such cat state. Possible solution of the Schrödinger's cat paradox is considered. We pointed out that the collapsed state of the cat always shows definite and predictable outcomes even if cat also consists of a superposition: $\mid$ cat $\rangle=c_{1} \mid$ live cat $\rangle+c_{2} \mid$ death cat $\rangle$.
\end{abstract}

\section{Keywords}

Probability Representation of Quantum States, Schrödinger's Cat, GRW

Collapse Model, Von Neumann Measurement Postulate

\section{Introduction}

As Weinberg recently reminded us [1], the measurement problem remains a fundamental conundrum. During measurement, the state vector of the microscopic system collapses in a probabilistic way to one of a number of classical states, 
in a way that is unexplained, and cannot be described by the time-dependent Schrödinger equation [1]. To review the essentials, it is sufficient to consider two-state systems. Suppose a nucleus $\boldsymbol{n}$, whose Hilbert space is spanned by orthonormal states $\left|s_{i}(t)\right\rangle, i=1,2$, where $\left|s_{1}(t)\right\rangle=\mid$ undecayed nucleus at instant $\left.t\right\rangle$ and $\left|s_{2}(t)\right\rangle=\mid$ decayed nucleus at instant $\left.t\right\rangle$ is in the superposition state,

$$
\left|\Psi_{t}\right\rangle_{n}=c_{1}\left|s_{1}(t)\right\rangle+c_{2}\left|s_{2}(t)\right\rangle,\left|c_{1}\right|^{2}+\left|c_{2}\right|^{2}=1 \text {. }
$$

A measurement apparatus $A$, which may be microscopic or macroscopic, is designed to distinguish between states $\left|s_{i}(t)\right\rangle$ by transitioning at each instant $t$ into state $\left|a_{i}(t)\right\rangle$ if it finds that $\boldsymbol{n}$ is in $\left|s_{i}(t)\right\rangle, i=1,2$. Assume that the detector is reliable, implying that the $\left|a_{1}(t)\right\rangle$ and $\left|a_{2}(t)\right\rangle$ are orthonormal at each instant $t$, i.e., $\left\langle a_{1}(t) \mid a_{2}(t)\right\rangle=0$ and that the measurement interaction does not disturb states $\left|s_{i}\right\rangle$-i.e., the measurement is "ideal". When $A$ measures $\left|\Psi_{t}\right\rangle_{n}$, the Schrödinger equation's unitary time evolution then leads to the "measurement state" $\left|\Psi_{t}\right\rangle_{n A}$ :

$$
\left|\Psi_{t}\right\rangle_{n A}=c_{1}\left|a_{1}(t)\right\rangle+c_{2}\left|a_{2}(t)\right\rangle,\left|c_{1}\right|^{2}+\left|c_{2}\right|^{2}=1
$$

of the composite system $\boldsymbol{n} A$ following the measurement.

Standard formalism of continuous quantum measurements [2] [3] [4] [5] leads to a definite but unpredictable measurement outcome, either $\left|a_{1}(t)\right\rangle$ or $\left|a_{2}(t)\right\rangle$ and that $\left|\Psi_{t}\right\rangle_{n}$ suddenly "collapses" at instant $t^{\prime}$ into the corresponding state $\left|s_{i}\left(t^{\prime}\right)\right\rangle$. But unfortunately Equation (1.2) does not appear to resemble such a collapsed state at instant $t^{\prime}$ ? .

The measurement problem is as follows:

(I) How do we reconcile the canonical collapse model that postulates [2] definite but unpredictable outcomes with the "measurement state" $\left|\Psi_{t}\right\rangle_{n A}$.

(II) How do we reconcile the measurement that postulates definite but unpredictable outcomes with the "measurement state" $\left|\Psi_{t}\right\rangle_{n A}$ at each instant $t$ and

(III) How does the outcome become irreversibly recorded in light of the Schrödinger equation's unitary and, hence, reversible evolution?

This paper deals with only the special case of the measurement problem, known as Schrödinger's cat paradox (Figure 1). For a good and complete explanation of this paradox one can see Leggett [6] and Hobson [7].

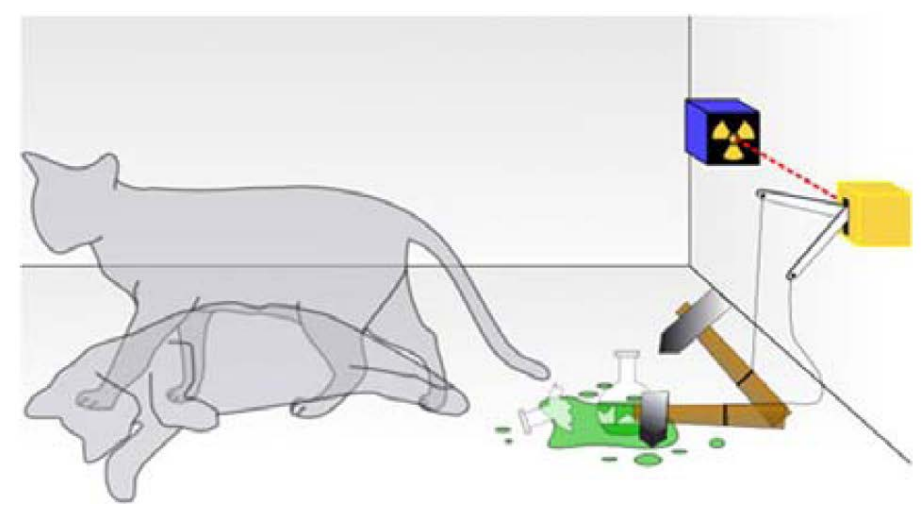

Figure 1. Schrödinger's cat adapted to the measurement of position of an alpha particle [8] [9] [10]. 
In his famous thought experiment [11], Schrôdinger (1935) imagined a cat that measures the value of a quantum mechanical observable with its life. Adapted to the measurement of position of an alpha particle, the experiment is this. A cat, a flask of poison, and a radioactive source are placed in a sealed box. If an internal monitor detects radioactivity (i.e. a single atom decaying), the flask is shattered, releasing the poison that kills the cat. The Copenhagen interpretation of quantum mechanics implies that after a while, the cat is simultaneously alive and dead. Yet, when one looks in the box, one sees the cat either alive or dead, not both alive and dead.

This poses the question of when exactly quantum superposition ends and reality collapses into one possibility or the other?

Since Schrödinger's time, no any interpretations or extensions of quantum mechanics have been proposed which gives clear unambiguous answers to the questions posed by Schrödinger's cat of how long superpositions last and when (or whether) they collapse.

\section{The canonical interpretations of the experiment \\ Copenhagen interpretation}

The most commonly held interpretation of quantum mechanics is the Copenhagen interpretation [12]. In the Copenhagen interpretation, a system stops being a superposition of states and becomes either one or the other when an observation takes place. This thought experiment makes apparent the fact that the nature of measurement, or observation, is not well-defined in this interpretation. The experiment can be interpreted to mean that while the box is closed, the system simultaneously exists in a superposition of the states "decayed nucleus/dead cat" and "undecayed nucleus/living cat", and that only when the box is opened and an observation performed does the wave function collapse into one of the two states.

However, one of the main scientists associated with the Copenhagen interpretation, Niels Bohr, never had in mind the observer-induced collapse of the wave function, so that Schrödinger's cat did not pose any riddle to him. The cat would be either dead or alive long before the box is opened by a conscious observer [13]. Analysis of an actual experiment found that measurement alone (for example by a Geiger counter) is sufficient to collapse a quantum wave function before there is any conscious observation of the measurement [14]. The view that the "observation" is taken when a particle from the nucleus hits the detector can be developed into objective collapse theories. The thought experiment requires an "unconscious observation" by the detector in order for magnification to occur.

\section{Objective collapse theories}

According to objective collapse theories, superpositions are destroyed spontaneously (irrespective of external observation) when some objective physical threshold (of time, mass, temperature, irreversibility, etc.) is reached. Thus, the cat would be expected to have settled into a definite state long before the box is opened. This could loosely be phrased as "the cat observes itself", or "the environment observes the cat". 
Objective collapse theories require a modification of standard quantum mechanics to allow superpositions to be destroyed by the process of time evolution. This process, known as "decoherence", is among the fastest processes currently known to physics [15].

\section{Ensemble interpretation}

The ensemble interpretation states that superpositions are nothing but subensembles of a larger statistical ensemble. The state vector would not apply to individual cat experiments, but only to the statistics of many similarly prepared cat experiments. Proponents of this interpretation state that this makes the Schrödinger's cat paradox a trivial matter, or a non-issue. This interpretation serves to discard the idea that a single physical system in quantum mechanics has a mathematical description that corresponds to it in any way.

Remark 1.1. Ensemble interpretation is in a good agreement with a canonical interpretation of the wave function ( $\psi$-function) in canonical QM-measurement theory. However under rigorous consideration of a dynamics of the Schrödinger's cat, this interpretation gives unphysical result (see Proposition 3.2. (ii)).

\section{The canonical collapse models}

In order to appreciate how canonical collapse models work, and what they are able to achieve, we briefly review the GRW model. Let us consider a system of $n$ particles which, only for the sake of simplicity, we take to be scalar and spinless; the GRW model is defined by the following postulates: (1) The state of the system is represented by a wave function $\psi_{t}\left(\boldsymbol{x}_{1}, \boldsymbol{x}_{2}, \cdots, \boldsymbol{x}_{n}\right)$ belonging to the Hilbert space $£_{2}\left(\mathbb{R}^{3 n}\right)$. (2) At random times, the wave function experiences a sudden jump of the form:

$$
\psi_{t}\left(\boldsymbol{x}_{1}, \boldsymbol{x}_{2}, \cdots, \boldsymbol{x}_{n}\right) \rightarrow \psi_{t}\left(\boldsymbol{x}_{1}, \boldsymbol{x}_{2}, \cdots, \boldsymbol{x}_{n} ; \tilde{\boldsymbol{x}}_{m}\right)=\frac{\mathfrak{R}_{m}\left(\tilde{\boldsymbol{x}}_{m}\right) \psi_{t}\left(\boldsymbol{x}_{1}, \boldsymbol{x}_{2}, \cdots, \boldsymbol{x}_{n}\right)}{\left\|\mathfrak{R}_{m}\left(\tilde{\boldsymbol{x}}_{m}\right) \psi_{t}\left(\boldsymbol{x}_{1}, \boldsymbol{x}_{2}, \cdots, \boldsymbol{x}_{n}\right)\right\|_{2}},
$$

where $\psi_{t}\left(\boldsymbol{x}_{1}, \boldsymbol{x}_{2}, \cdots, \boldsymbol{x}_{n}\right)$ is the state vector of the whole system at time $t$, immediately prior to the jump process and $\mathfrak{R}_{n}\left(\tilde{\boldsymbol{x}}_{m}\right)$ is a linear operator which is conventionally chosen equal to:

$$
\mathfrak{R}_{m}\left(\tilde{\boldsymbol{x}}_{m}\right)=\left(\pi r_{c}^{2}\right)^{-3 / 4} \exp \left[-\frac{\left(\hat{\boldsymbol{x}}_{m}-\tilde{\boldsymbol{x}}_{m}\right)^{2}}{2 r_{c}^{2}}\right]
$$

where $r_{c}$ is a new parameter of the model which sets the width of the localization process, and $\hat{\boldsymbol{x}}_{m}$ is the position operator associated to the $m$-th particle of the system and the random variable $\hat{\boldsymbol{x}}_{m}$ corresponds to the place where the jump occurs. (3) It is assumed that the jumps are distributed in time like a Poissonian process with frequency $\lambda=\lambda_{\mathrm{GRW}}$ this is the second new parameter of the model. (4) Between two consecutive jumps, the state vector evolves according to the standard Schrödinger equation.

The 1-particle master equation of the GRW model takes the form

$$
\frac{\mathrm{d}}{\mathrm{d} t} \rho(t)=-\frac{i}{\hbar}[\hat{\boldsymbol{H}}, \rho(t)]-T[\rho(t)] \text {. }
$$

Here $\hat{\boldsymbol{H}}$ is the standard quantum Hamiltonian of the particle, and $T[\cdot]$ 
represents the effect of the spontaneous collapses on the particle's wave function. In the position representation, this operator becomes:

$$
\langle\boldsymbol{x}|T[\rho(t)]| \boldsymbol{y}\rangle=\lambda\left\{1-\exp \left[-\frac{(\boldsymbol{x}-\boldsymbol{y})^{2}}{4 r_{c}^{2}}\right]\right\}\langle\boldsymbol{x}|\rho(t)| \boldsymbol{y}\rangle .
$$

Another modern approach to stochastic reduction is to describe it using a stochastic nonlinear Schrödinger equation, an elegant simplied example of which is the following one particle case known as Quantum Mechanics with Universal Position Localization [QMUPL]:

$\mathrm{d}\left|\psi_{t}(x)\right\rangle=\left[-\frac{i}{\hbar} \hat{\boldsymbol{H}}-k\left(\hat{q}-\left\langle q_{t}\right\rangle\right)^{2} \mathrm{~d} t\right]\left|\psi_{t}(x)\right\rangle \mathrm{d} t+\sqrt{2 k}\left(\hat{q}-\left\langle q_{t}\right\rangle\right) \mathrm{d} W_{t}\left|\psi_{t}(x)\right\rangle$.

Here $\hat{q}$ is the position operator, $\left\langle q_{t}\right\rangle=\left\langle\psi_{t}|\hat{q}| \psi_{t}\right\rangle$ it is its expectation value, and $k$ is a constant, characteristic of the model, which sets the strength of the collapse mechanics, and it is chosen proportional to the mass $m$ of the particle according to the formula: $k=\left(\mathrm{m} / m_{0}\right) \lambda_{0}$, where $m_{0}$ is the nucleon's mass and $\lambda_{0}$ measures the collapse strength. It is easy to see that Equation (1.5) contains both non-linear and stochastic terms, which are necessary to induce the collapse of the wave function. For example let us consider a free particle $\left(\hat{\boldsymbol{H}}=p^{2} / 2 m\right)$, and a Gaussian state:

$$
\psi_{t}(x)=\exp \left\{-a_{t}\left(x-\bar{x}_{t}\right)^{2}+i \bar{k}_{t} x\right\} .
$$

It is easy to see that $\psi_{t}(x)$ given by Equation (1.6) is solution of Equation (1.5), where

$$
\frac{\mathrm{d} a_{t}}{\mathrm{~d} t}=k-\frac{2 i \hbar}{m} a_{t}^{2}, \frac{\mathrm{d} \bar{x}_{t}}{\mathrm{~d} t}=\frac{\hbar}{m} \bar{k}_{t}+\frac{\sqrt{k}}{2 \operatorname{Re}\left(a_{t}\right)} \dot{W}_{t}, \frac{\mathrm{d} \bar{k}_{t}}{\mathrm{~d} t}=-\sqrt{k} \frac{\operatorname{Im}\left(a_{t}\right)}{\operatorname{Re}\left(a_{t}\right)} \dot{W}_{t}
$$

The CSL model is defined by the following stochastic differential equation in the Fock space:

$$
\begin{aligned}
\mathrm{d}\left|\psi_{t}(\boldsymbol{x})\right\rangle= & {\left[-\frac{i}{\hbar} \hat{\boldsymbol{H}}-k\left(\hat{M}(\boldsymbol{x})-\left\langle M_{t}(\boldsymbol{x})\right\rangle\right)^{2} \mathrm{~d} t\right]\left|\psi_{t}(\boldsymbol{x})\right\rangle \mathrm{d} t } \\
& +\sqrt{2 k}\left(\hat{M}(\boldsymbol{x})-\left\langle M_{t}(\boldsymbol{x})\right\rangle\right) \mathrm{d} W_{t}(\boldsymbol{x})\left|\psi_{t}(\boldsymbol{x})\right\rangle .
\end{aligned}
$$

\section{Generalized Gamov Theory of the Alpha Decay via Tunneling Using GRW Collapse Model}

By 1928, George Gamow had solved the theory of the alpha decay via tunneling [8]. The alpha particle is trapped in a potential well by the nucleus. Classically, it is forbidden to escape, but according to the (then) newly discovered principles of quantum mechanics, it has a tiny (but non-zero) probability of "tunneling" through the barrier and appearing on the other side to escape the nucleus. Gamow solved a model potential for the nucleus and derived, from first principles, a relationship between the half-life of the decay, and the energy of the emission.

The $\alpha$-particle has total energy $E$ and is incident on the barrier from the right to left. 


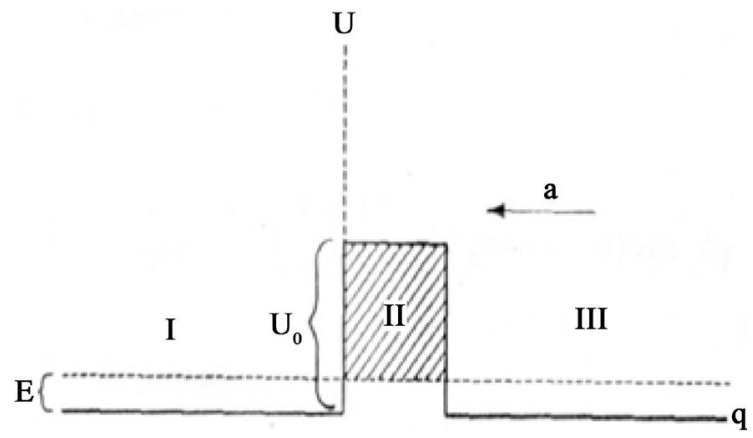

Figure 2. The particle has total energy $E$ and is incident on the barrier $V(x)$ from right to left. Adapted from [8].

The Schrödinger equation in each of regions $\mathbf{I}=\{x \mid x<0\}, \mathbf{I I}=\{x \mid 0 \leq x \leq l\}$ and $\mathbf{I I I}=\{x \mid x>l\}$ takes the following form

$$
\frac{\partial^{2} \Psi(x)}{\partial x^{2}}+\frac{2 m}{\hbar^{2}}[E-U(x)] \Psi(x)=0,
$$

where

$$
U(x)=\left\{\begin{array}{l}
0 \text { for } x<0 \\
U_{0} \text { for } 0 \leq x \leq l \\
0 \text { for } x>l
\end{array}\right.
$$

The solutions read [8]:

$$
\begin{gathered}
\Psi_{\mathrm{III}}(x)=C_{+} \exp (i k x)+C_{-} \exp (-i k x), \\
\Psi_{\mathrm{II}}(x)=B_{+} \exp \left(k^{\prime} x\right)+B_{-} \exp \left(-k^{\prime} x\right), \\
\Psi_{\mathrm{I}}(x)=A \cos (k x) \\
=\frac{A}{2}[\exp (i k x)+\exp (-i k x)],
\end{gathered}
$$

where

$$
\begin{aligned}
& k=\frac{2 \pi}{\hbar} \sqrt{2 m E}, \\
& k^{\prime}=\frac{2 \pi}{\hbar} \sqrt{2 m\left(U_{0}-E\right)} .
\end{aligned}
$$

At the boundary $x=0$ we have the following boundary conditions:

$$
\begin{gathered}
\left.\Psi_{\mathbf{I}}(0)\right|_{x=0}=\left.\Psi_{\mathbf{I I}}(0)\right|_{x=0}, \\
\left.\frac{\partial \Psi_{\mathbf{I}}(x)}{\partial x}\right|_{x=0}=\left.\frac{\partial \Psi_{\mathbf{I I}}(x)}{\partial x}\right|_{x=0} .
\end{gathered}
$$

At the boundary $x=l$ we have the following boundary conditions

$$
\begin{gathered}
\left.\Psi_{\text {II }}(l)\right|_{x=l}=\left.\Psi_{\text {III }}(l)\right|_{x=l}, \\
\left.\frac{\partial \Psi_{\text {II }}(x)}{\partial x}\right|_{x=l}=\left.\frac{\partial \Psi_{\text {III }}(x)}{\partial x}\right|_{x=l} .
\end{gathered}
$$

From the boundary conditions (2.5)-(2.6) one obtains [8]: 


$$
\begin{aligned}
& B_{+}=\frac{A}{2}\left(1+i \frac{k}{k^{\prime}}\right), B_{-}=\frac{A}{2}\left(1-i \frac{k}{k^{\prime}}\right), \\
& C_{+}=A\left[\operatorname{ch}\left(k^{\prime} l\right)+i D \operatorname{sh}\left(k^{\prime} l\right)\right], C_{-}=i\left(A S \operatorname{sh}\left(k^{\prime} l\right) \exp (i k l)\right), \\
& D=\frac{1}{2}\left(\frac{k}{k^{\prime}}-\frac{k^{\prime}}{k}\right), S=\frac{1}{2}\left(\frac{k}{k^{\prime}}+\frac{k^{\prime}}{k}\right) .
\end{aligned}
$$

From (2.7) one obtain the conservation law

$$
|A|^{2}=\left|C_{+}\right|^{2}-\left|C_{-}\right|^{2} \text {. }
$$

Let us introduce now a function $E_{\mathbf{I I}}(x, l)=\theta_{2}(x, l) E_{2}(x, l)$ where

$$
\begin{aligned}
& E_{2}(x, l)=\left\{\begin{array}{l}
\left(\pi r_{c}^{2}\right)^{-1 / 4} \exp \left(-\frac{x^{2}}{2 r_{c}^{2}}\right) \text { for }-\infty<x<\frac{l}{2} \\
\left(\pi r_{c}^{2}\right)^{-1 / 4} \exp \left(-\frac{(x-l)^{2}}{2 r_{c}^{2}}\right) \text { for } \frac{l}{2} \leq x<\infty
\end{array}\right. \\
& \theta_{2}(x, l)=\left\{\begin{array}{l}
1 \text { for } x \in[0, l] \\
0 \text { for } x \notin[0, l]
\end{array}\right.
\end{aligned}
$$

Assumption 2.1. We assume now that:

(i) at instant $t=0$ the wave function $\Psi_{\mathbf{I}}(x)$ experiences a sudden jump of the form

$$
\Psi_{\mathbf{I}}(x) \rightarrow \Psi_{\mathbf{I}}^{\#}(x)=\frac{\mathfrak{R}_{\mathbf{I}}(\hat{x}) \Psi_{\mathbf{I}}(x)}{\left\|\Re_{\mathbf{I}}(\hat{x}) \Psi_{\mathbf{I}}(x)\right\|_{2}},
$$

where $\mathfrak{R}_{\mathbf{I}}(\hat{x})$ is a linear operator which is chosen equal to:

$$
\mathfrak{R}_{\mathbf{I}}(\hat{x})=\left(\pi r_{c}^{2}\right)^{-1 / 4} \theta_{1}(\hat{x}, l) \exp \left[-\frac{\hat{x}^{2}}{2 r_{c}^{2}}\right] ;
$$

where

$$
\theta_{1}(x, l)=\left\{\begin{array}{l}
1 \text { for } x \in[-l, 0] \\
0 \text { for } x \notin[-l, 0]
\end{array}\right.
$$

Remark 2.1. Note that: $\operatorname{supp}\left(\Psi_{\mathbf{I}}^{\#}(x)\right) \subseteq[-l, 0]$.

(ii) at instant $t=0$ the wave function $\Psi_{\text {II }}(x)$ experiences a sudden jump of the form

$$
\Psi_{\mathrm{II}}(x) \rightarrow \Psi_{\mathrm{II}}^{\#}(x)=\frac{\mathfrak{R}_{\mathrm{II}}(\hat{x}) \Psi_{\mathrm{II}}(x)}{\left\|\Re_{\mathrm{II}}(\hat{x}) \Psi_{\mathrm{II}}(x)\right\|_{2}},
$$

where $\mathfrak{R}_{\mathbf{I I}}(\hat{x})$ is a linear operator which is chosen equal to:

$$
\mathfrak{R}_{\mathbf{I I}}(\hat{x})=E_{\mathbf{I I}}(\hat{x}, l) ;
$$

Remark 2.2. Note that: $\operatorname{supp}\left(\Psi_{\text {II }}^{\#}(x)\right) \subseteq[0, l]$.

(iii) at instant $t=0$ the wave function $\Psi_{\text {III }}(x)$ experiences a sudden jump of the form

$$
\Psi_{\text {III }}(x) \rightarrow \Psi_{\text {III }}^{\#}(x)=\frac{\Re_{\text {III }}(\hat{x}) \Psi_{\text {III }}(x)}{\left\|\Re_{\text {III }}(\hat{x}) \Psi_{\text {III }}(x)\right\|_{2}},
$$

where $\mathfrak{R}_{\text {III }}(\hat{x})$ is a linear operator which is chosen equal to: 


$$
\mathfrak{R}_{\text {III }}(\hat{x})=\left(\pi r_{c}^{2}\right)^{-1 / 4} \exp \left[-\frac{(\hat{x}-l)^{2}}{2 r_{c}^{2}}\right] .
$$

Remark 2.3. Note that we have chosen operators (2.10), (2.12) and (2.14) such that the boundary conditions (2.5), (2.6) are satisfied.

Definition 2.1. Let $\Psi(x)$ be a solution of the Schrödinger Equation (2.1). The stationary Schrödinger Equation (2.1) is a weakly well preserved in region $\Gamma \subseteq \mathbb{R}$ by collapsed wave function $\Psi^{\#}(x)$ if there exist an wave function $\Psi(x)$ such that the estimate

$$
\int_{\Gamma}\left\{\frac{\partial^{2} \Psi^{\#}(x)}{\partial x^{2}}+\frac{2 m}{\hbar^{2}}[E-U(x)] \Psi^{\#}(x)\right\} d x=O\left(\hbar^{2+\alpha}\right),
$$

where $\alpha \geq 1$, is satisfied.

Proposition 2.1. The Schrödinger equation in each of regions I, II, III is a weakly well preserved by collapsed wave function $\Psi_{\mathrm{I}}^{\#}(x), \Psi_{\mathrm{II}}^{\#}(x)$ and $\Psi_{\mathrm{III}}^{\#}(x)$ correspondingly.

Proof. See Appendix B.

Definition 2.2. Let us consider the time-dependent Schrödinger equation:

$$
\begin{aligned}
& i \hbar \frac{\partial \Psi(\boldsymbol{x}, t)}{\partial t}=\hat{\boldsymbol{H}} \Psi(\boldsymbol{x}, t) \\
& t \in[0, T], \boldsymbol{x} \in \mathbb{R}^{3 n} .
\end{aligned}
$$

The time-dependent Schrödinger Equation (2.16) is a weakly well preserved by corresponding to $\Psi(x, t)$ collapsed wave function $\Psi^{\#}(x, t)$

$$
\begin{aligned}
\Psi^{\#}\left(\boldsymbol{x}_{1}, \boldsymbol{x}_{2}, \cdots, \boldsymbol{x}_{n}, t\right) & =\Psi\left(\boldsymbol{x}_{1}, \boldsymbol{x}_{2}, \cdots, \boldsymbol{x}_{n}, t ; \tilde{\boldsymbol{x}}_{m_{1}}, \cdots, \tilde{\boldsymbol{x}}_{m_{k}}\right) \\
& =\frac{\Re_{m_{1}, \cdots, m_{k}}\left(\tilde{\boldsymbol{x}}_{m_{1}}, \cdots, \tilde{\boldsymbol{x}}_{m_{k}}\right) \Psi\left(\boldsymbol{x}_{1}, \boldsymbol{x}_{2}, \cdots, \boldsymbol{x}_{n}, t\right)}{\left\|\Re_{m_{1}, \cdots, m_{k}}\left(\tilde{\boldsymbol{x}}_{m_{1}}, \cdots, \tilde{\boldsymbol{x}}_{m_{k}}\right) \Psi\left(\boldsymbol{x}_{1}, \boldsymbol{x}_{2}, \cdots, \boldsymbol{x}_{n}, t\right)\right\|_{2}}, \\
\mathfrak{R}_{m_{1}, \cdots, m_{k}}\left(\tilde{\boldsymbol{x}}_{m_{1}}, \cdots, \tilde{\boldsymbol{x}}_{m_{k}}\right) & =\prod_{i=1}^{k} \Re_{m_{i}}\left(\tilde{\boldsymbol{x}}_{m_{i}}\right)
\end{aligned}
$$

in region $\Gamma \subseteq \mathbb{R}^{3 d}$ if there exist an wave function $\Psi(x, t)$ such that the estimate

$$
\begin{aligned}
& \int_{\Gamma}\left\{i \hbar \frac{\partial \Psi^{\#}(\boldsymbol{x}, t)}{\partial t}-\hat{\boldsymbol{H}} \Psi^{\#}(\boldsymbol{x}, t)\right\} d^{3 d} x=O\left(\hbar^{\alpha}\right), \\
& t \in[0, T], \boldsymbol{x} \in \mathbb{R}^{3 d}
\end{aligned}
$$

where $\alpha \geq 1$, is satisfied.

Definition 2.3. Let $\Psi^{\#}(x, t)=\Psi^{\#}\left(x_{1}, x_{2}, \cdots, x_{d}, t\right)$ be a function $\Psi\left(\boldsymbol{x}_{1}, \boldsymbol{x}_{2}, \cdots, \boldsymbol{x}_{d}, t ; \tilde{\boldsymbol{x}}_{1}, \cdots, \tilde{\boldsymbol{x}}_{d}\right)$. Let us consider the Probability Current Law

$$
\begin{aligned}
& \frac{\partial}{\partial t} P(\Gamma, t)+\int_{\partial \Gamma} \boldsymbol{J}\left(\boldsymbol{x}_{1}, \boldsymbol{x}_{2}, \cdots, \boldsymbol{x}_{d}, t\right) \cdot \boldsymbol{n d}^{2 d} x=O\left(\hbar^{\alpha}\right), \\
& \boldsymbol{J}\left(\boldsymbol{x}_{1}, \boldsymbol{x}_{2}, \cdots, \boldsymbol{x}_{d}, t\right)=\Psi(\boldsymbol{x}, t) \nabla \overline{\Psi(\boldsymbol{x}, t)}-\overline{\Psi(\boldsymbol{x}, t)} \nabla \Psi(\boldsymbol{x}, t), \\
& t \in[0, T], \boldsymbol{x} \in \mathbb{R}^{3 d},
\end{aligned}
$$

corresponding to Schrödinger Equation (2.16). Probability Current Law (2.18) is a weakly well preserved by corresponding to $\Psi(\boldsymbol{x}, t)$ collapsed wave function 
$\Psi^{\#}(\boldsymbol{x}, t)$ in region $\Gamma \subseteq \mathbb{R}^{3 d}$ if there exist an wave function $\Psi(\boldsymbol{x}, t)$ such that the estimate

$$
\begin{aligned}
& \frac{\partial}{\partial t} P(\Gamma, t)+\int_{\partial \Gamma} \boldsymbol{J}^{\#}\left(\boldsymbol{x}_{1}, \boldsymbol{x}_{2}, \cdots, \boldsymbol{x}_{d}, t\right) \cdot \boldsymbol{n} \mathrm{d}^{2 d} x=O\left(\hbar^{\alpha}\right), \\
& \begin{aligned}
\boldsymbol{J}^{\#}\left(\boldsymbol{x}_{1}, \boldsymbol{x}_{2}, \cdots, \boldsymbol{x}_{d}, t\right) & =\Psi^{\#}(\boldsymbol{x}, t) \nabla \overline{\Psi^{\#}(\boldsymbol{x}, t)}-\overline{\Psi^{\#}(\boldsymbol{x}, t)} \nabla \Psi^{\#}(\boldsymbol{x}, t) \\
= & O\left(\hbar^{\alpha}\right), \\
& t \in[0, T], \boldsymbol{x} \in \mathbb{R}^{3 d},
\end{aligned}
\end{aligned}
$$

where $\alpha \geq 1$, is satisfied.

Proposition 2.2. Assume that there exists an wave function $\Psi(x, t)$ such that the estimate (2.17) is satisfied. Then Probability Current Law (2.18) is a weakly well preserved by corresponding to $\Psi(x, t)$ collapsed wave function $\Psi^{\#}(x, t)$ in region $\Gamma \subseteq \mathbb{R}^{3 d}$, i.e. the estimate (2.19) is satisfied on the wave function $\Psi^{\#}(x, t)$.

\section{Schrödinger's Cat Paradox Resolution}

In this section we shall consider the problem of the collapse of the cat state vector on the basis of two different hypotheses:

(A) The canonical postulate of QM is correct in all cases.

(B) The canonical interpretation of the wave function $\psi=c_{1} \psi_{1}+c_{2} \psi_{2}$ is correct only when the supports the wave functions $\psi_{1}$ and $\psi_{2}$ essentially overlap. When the wave functions $\psi_{1}$ and $\psi_{2}$ have separated supports (as in the case of the experiment that we are considering in section II) we claim that canonical interpretation of the wave function $\psi=c_{1} \psi_{1}+c_{2} \psi_{2}$ is no longer valid for a such cat state (for details see Appendix C).

\subsection{Consideration of the Schrödinger's Cat Paradox Using Canonical Von Neumann Postulate}

Let $\left|s_{1}(t)\right\rangle$ and $\left|s_{2}(t)\right\rangle$ be

$$
\begin{aligned}
& \left.\left|s_{1}(t)\right\rangle=\mid \text { undecayed nucleus at instant } t\right\rangle, \\
& \left.\left|s_{2}(t)\right\rangle=\mid \text { decayed nucleus at instant } t\right\rangle .
\end{aligned}
$$

In a good approximation we assume now that

$$
\left|s_{1}(0)\right\rangle=\int_{-\infty}^{+\infty} \Psi_{\text {II }}^{\#}(x)|x\rangle \mathrm{d} x
$$

and

$$
\left|s_{2}(0)\right\rangle=\int_{-\infty}^{+\infty} \Psi_{\mathbf{I}}^{\#}(x)|x\rangle \mathrm{d} x .
$$

Remark 3.1. Note that:

(i) $\left|s_{2}(0)\right\rangle=\mid$ decayed nucleus at instant 0$\rangle=\mid$ free $\alpha$-particle at instant 0$\rangle$.

(ii) Feynman propagator of a free $\alpha$-particle is [9]:

$$
K_{2}\left(x, t, x_{0}\right)=\left(\frac{m}{2 \pi i \hbar t}\right)^{1 / 2} \exp \left\{\frac{i}{\hbar}\left[\frac{m\left(x-x_{0}\right)^{2}}{2 t}\right]\right\} .
$$


Therefore from Equations ((3.3), (2.9) and (3.4)) we obtain

$$
\begin{aligned}
\left|s_{2}(t)\right\rangle= & \int_{-\infty}^{+\infty} \Psi_{\mathbf{I}}^{\#}(x, t)|x\rangle \mathrm{d} x, \\
\Psi_{\mathbf{I}}^{\#}(x, t)= & \int_{-\infty}^{0} \Psi_{\mathbf{I}}^{\#}\left(x_{0}\right) K_{2}\left(x, t, x_{0}\right) \mathrm{d} x_{0} \\
= & \left(\pi r_{c}^{2}\right)^{-1 / 4} \times\left(\frac{m}{2 \pi i \hbar t}\right)^{1 / 2} \times \int_{-\infty}^{0} \theta_{1}\left(x_{0}, l\right) \exp \left(-\frac{x_{0}^{2}}{2 r_{c}^{2}}\right) \exp \left(-i \frac{2 \pi}{\hbar} \sqrt{2 m E} x_{0}\right) \\
& \times \exp \left\{\frac{i}{\hbar}\left[\frac{m\left(x-x_{0}\right)^{2}}{2 t}\right]\right\} \mathrm{d} x_{0} \\
= & \left(\pi r_{c}^{2}\right)^{-1 / 4} \times\left(\frac{m}{2 \pi i \hbar}\right)^{1 / 2} \times \int_{-l}^{0} \theta_{1}\left(x_{0}, l\right) \exp \left(-\frac{x_{0}^{2}}{2 r_{c}^{2}}\right) \\
& \times \exp \left\{\frac{i}{\hbar}\left[\frac{m\left(x-x_{0}\right)^{2}}{2 t}-\pi \sqrt{4 m E} x_{0}\right]\right\} \mathrm{d} x_{0} \\
= & \left(\pi r_{c}^{2}\right)^{-1 / 4} \times\left(\frac{m}{2 \pi i \hbar t}\right)^{1 / 2} \times \int_{-l}^{0} \theta_{1}\left(x_{0}, l\right) \exp \left(-\frac{x_{0}^{2}}{2 r_{c}^{2}}\right) \times \exp \left\{\frac{i}{\hbar}\left[S\left(t, x, x_{0}\right)\right]\right\} \mathrm{d} x_{0},
\end{aligned}
$$

where

$$
S\left(t, x, x_{0}\right)=\frac{m\left(x-x_{0}\right)^{2}}{2 t}-\pi \sqrt{8 m E} x_{0} .
$$

We assume now that

$$
\hbar \ll 2 r_{c}^{2} \ll l^{2}<1 .
$$

Oscillatory integral in RHS of Equation (3.5) is calculated now directly using stationary phase approximation. The phase term $S\left(x, x_{0}\right)$ given by Equation (3.6) is stationary when

$$
\frac{\partial S\left(t, x, x_{0}\right)}{\partial x_{0}}=-\frac{m\left(x-x_{0}\right)}{t}-\pi \sqrt{8 m E}=0 .
$$

Therefore

$$
\begin{aligned}
-\frac{m\left(x-x_{0}\right)}{t}-\pi \sqrt{8 m E} & =0, \\
-\left(x-x_{0}\right) & =\pi t \sqrt{8 E / m},
\end{aligned}
$$

and thus stationary point $x_{0}(t, x)$ are

$$
x_{0}(t, x)=\pi t \sqrt{8 E / m}+x .
$$

Thus from Equation (3.5) and Equation (3.10) using stationary phase approximation we obtain

$$
\begin{aligned}
\left|s_{2}(t)\right\rangle= & \int_{-\infty}^{+\infty} \Psi_{\mathrm{I}}^{\#}(x, t)|x\rangle \mathrm{d} x, \\
\Psi_{\mathrm{I}}^{\#}(x, t)= & \left(\pi r_{c}^{2}\right)^{-1 / 4} \times \theta_{1}\left(x_{0}(t, x), l\right) \exp \left[-\frac{x_{0}^{2}(t, x)}{2 r_{c}^{2}}\right] \\
& \times \exp \left\{\frac{i}{\hbar}\left[S\left(t, x, x_{0}(t, x)\right)\right]\right\}+O(\hbar),
\end{aligned}
$$


where

$$
S\left(x, x_{0}(t, x)\right)=\frac{m\left(x-x_{0}(t, x)\right)^{2}}{2 t}-\pi \sqrt{8 m E} x_{0}(t, x) .
$$

From Equation (3.10) we obtain

$$
\overline{\Psi_{\mathrm{I}}^{\#}(x, t)} \Psi_{\mathrm{I}}^{\#}(x, t) \simeq\left(\pi r_{c}^{2}\right)^{-1 / 2} \times \theta_{1}(x+\pi t \sqrt{8 E / m}, l) \exp \left[-\frac{(x+\pi t \sqrt{8 E / m})^{2}}{r_{c}^{2}}\right] \text {. }
$$

Remark 3.2. From the inequality (3.7) and Equation (3.13) follows that $\alpha$ -particle at each instant $t \geq 0$ moves quasiclassically from right to left by the law

$$
x(t)=-\pi t \sqrt{8 E / m},
$$

i.e., estimating the position $x\left(t, x_{0}, t_{0} ; \hbar\right)$ at each instant $t \geq 0$ with final error $r_{c}$ gives $|\langle x\rangle(t)-x(t)| \leq r_{c}, i=1, \cdots, d$ with a probability

$$
\boldsymbol{P}\left\{|\langle x\rangle(t, 0,0 ; \hbar)-x(t)| \leq r_{c}\right\}=1 .
$$

Remark 3.3. We assume now that a distance between radioactive source and internal monitor which detects a single atom decaying (see Figure 1) is equal to $L$.

Proposition 3.1. After $\alpha$-decay at instant $t=0$ the collaps: $\mid$ live cat $\rangle \rightarrow \mid$ death cat $\rangle$ arises at instant

$$
T=\frac{L}{\pi \sqrt{8 E / m}}
$$

with a probability $\boldsymbol{P}_{T}(\mid$ death cat $\left.\rangle\right)$ to observe a state $\mid$ death cat $\rangle$ at instant $T$ is $\boldsymbol{P}_{T}(\mid$ death cat $\left.\rangle\right)=1$.

Proof. Note that. In this case Schrödinger's cat in fact performs the single measurement of $\alpha$-particle position with accuracy of $\delta x=l$ at instant $t=T$ (given by Equation (3.15)) by internal monitor (see Figure 1). The probability of getting a result $L$ with accuracy of $\delta x=l$ given by

$$
\int_{|L-x| \leq I / 2}\left|\left\langle x \mid s_{2}(T)\right\rangle\right|^{2} \mathrm{~d} x=1 \text {. }
$$

Therefore at instant $T$ the $\alpha$-particle kills Schrödinger's cat with a probability $\boldsymbol{P}_{T}(\mid$ death cat $\left.\rangle\right)=1$.

Remark 3.4. Note that. When Schrödinger's cat has performed this measurement the immediate post measurement state of $\alpha$-particle (by von Neumann postulate C.4) will end up in the state

$$
\begin{aligned}
\left|\Psi_{T}\right\rangle & =\frac{\int_{|L-x| \leq l / 2}|x\rangle\left\langle x \mid s_{2}(T)\right\rangle \mathrm{d} x}{\sqrt{\left.\int_{|L-x| \leq I / 2}|x| s_{2}(T)\right\rangle\left.\right|^{2} \mathrm{~d} x}} \\
& =\int_{|L-x| \leq l / 2}|x\rangle\left\langle x \mid s_{2}(T)\right\rangle \mathrm{d} x \in \boldsymbol{S}_{\Theta}, \\
\Theta & =\{x|| L-x \mid \leq l / 2\} .
\end{aligned}
$$

From Equation (3.17) one obtains 


$$
\begin{aligned}
x^{\prime}\left|\Psi_{T}\right\rangle & =\int_{|L-x| \leq I / 2}\left\langle x^{\prime} \mid x\right\rangle\left\langle x \mid s_{2}(T)\right\rangle \mathrm{d} x \\
& =\int_{|L-x| \leq I / 2} \delta\left(x^{\prime}-x\right)\left\langle x \mid s_{2}(T)\right\rangle \mathrm{d} x=\Psi_{\mathbf{I}}^{\#}\left(x^{\prime}, t\right) .
\end{aligned}
$$

Therefore the state $\left|\Psi_{T}\right\rangle$ again kills Schrödinger's cat with a probability $\boldsymbol{P}_{T}(\mid$ death cat $\left.\rangle\right)=1$.

Suppose now that a nucleus $\boldsymbol{n}$, whose Hilbert space is spanned by orthonormal states $\left|s_{i}(t)\right\rangle, i=1,2$, where $\left|s_{1}(t)\right\rangle=\mid$ undecayed nucleus at instant $\left.t\right\rangle$ and $\left|s_{2}(t)\right\rangle=\mid$ decayed nucleus at instant $\left.t\right\rangle$ is in the superposition state

$$
\left|\Psi_{t}\right\rangle_{n}=c_{1}\left|s_{1}(t)\right\rangle+c_{2}\left|s_{2}(t)\right\rangle,\left|c_{1}\right|^{2}+\left|c_{2}\right|^{2}=1
$$

Remark 3.5. Note that: (i)

$\left|s_{1}(0)\right\rangle=\mid$ undecayed nucleus at instant $\left.t=0\right\rangle$

$=\mid \alpha$-particle iside region $(0, l]$ ati nstant $t=0\rangle$.

(ii) Feynman propagator of $\alpha$-particle inside region $(0,1]$ are [9]:

$$
K_{2}\left(x, t, x_{0}\right)=\left(\frac{m}{2 \pi i \hbar t}\right)^{1 / 2} \exp \left\{\frac{i}{\hbar}\left[S\left(t, x, x_{0}\right)\right]\right\},
$$

where

$$
S\left(t, x, x_{0}\right)=\frac{m\left(x-x_{0}\right)^{2}}{2 t}+m t\left(U_{0}-E\right) .
$$

Therefore from Equations ((2.11), (2.12) and (3.20), (3.21)) we obtain

$$
\begin{aligned}
\left|s_{1}(t)\right\rangle & =\int_{-\infty}^{+\infty} \Psi_{\mathrm{II}}^{\#}(x, t)|x\rangle \mathrm{d} x \\
\Psi_{\mathrm{II}}^{\#}(x, t) & =\int_{0}^{l} \Psi_{\mathrm{II}}^{\#}\left(x_{0}\right) K_{2}\left(x, t, x_{0}\right) \mathrm{d} x_{0} \\
& =\left(\frac{m}{2 \pi i \hbar t}\right)^{1 / 2} \int_{0}^{l} E\left(x_{0}, l\right) \Psi_{\mathrm{II}}\left(x_{0}\right) \theta_{l}\left(x_{0}\right) \exp \left\{\frac{i}{\hbar}\left[S\left(t, x, x_{0}\right)\right]\right\} \mathrm{d} x_{0},
\end{aligned}
$$

where

$$
\theta_{l}(x)=\left\{\begin{array}{lll}
1 & \text { for } & x \in[0, l] \\
0 & \text { for } & x \notin[0, l]
\end{array}\right.
$$

Remark 3.6. We assume for simplification now that

$$
k^{\prime} \leq 1 .
$$

Therefore oscillatory integral in RHS of Equation (3.22) is calculated now directly using stationary phase approximation. The phase term $S\left(x, x_{0}\right)$ given by Equation (3.21) is stationary when

$$
\frac{\partial S\left(t, x, x_{0}\right)}{\partial x_{0}}=-\frac{m\left(x-x_{0}\right)}{t}=0 .
$$

and thus stationary point $x_{0}(t, x)$ are

$$
\begin{aligned}
& -x+x_{0}=0 \\
& x_{0}(t, x)=x .
\end{aligned}
$$

Thus from Equation (3.22) and Equation (3.25) using stationary phase ap- 
proximation we obtain

$$
\begin{aligned}
& \Psi_{\mathrm{II}}^{\#}(x, t) \\
= & E\left(x_{0}(t, x), l\right) \Psi_{\mathrm{II}}\left(x_{0}(t, x)\right) \theta_{l}\left(x_{0}(t, x)\right) \exp \left\{\frac{i}{\hbar}\left[S\left(t, x, x_{0}(t, x)\right)\right]\right\}+O(\hbar) \\
= & E(x, l) \Psi_{\mathrm{II}}(x) \theta_{l}(x) \exp \left\{\frac{i}{\hbar}\left[m t\left(U_{0}-E\right)\right]\right\}+O(\hbar) \\
= & E(x, l) \theta_{l}(x) O(1) \exp \left\{\frac{i}{\hbar}\left[m t\left(U_{0}-E\right)\right]\right\}+O(\hbar) .
\end{aligned}
$$

Therefore from Equation (3.22) and Equation (3.26) we obtain

$$
\left|\Psi_{\mathrm{II}}^{\#}(x, t)\right|^{2}=E^{2}(x, l) \theta_{l}(x) O(1)+O(\hbar) .
$$

Remark 3.7. Note that for each instant $t>0: \operatorname{supp}\left(\Psi_{\text {II }}^{\#}(x, t)\right) \cap \operatorname{supp}\left(\Psi_{\mathrm{I}}^{\#}(x, t)\right)=\varnothing$.

Remark 3.8. Note that, from Equations ((3.11), (3.13), (3.19), (3.22)-(3.27) and (A.13)) by Remark 3.7 we obtain

$$
\begin{aligned}
{ }_{n}\left\langle\Psi_{t}|\hat{x}| \Psi_{t}\right\rangle_{n}= & \left|c_{1}\right|^{2}\left\langle s_{1}(t)|\hat{x}| s_{1}(t)\right\rangle+\left|c_{2}\right|^{2}\left\langle s_{2}(t)|\hat{x}| s_{2}(t)\right\rangle \\
& +c_{1} c_{2}^{*}\left\langle s_{2}(t)|\hat{x}| s_{1}(t)\right\rangle+c_{1}^{*} c_{2}\left\langle s_{2}(t)|\hat{x}| s_{1}(t)\right\rangle^{*} \\
= & \left|c_{1}\right|^{2}\left\langle s_{1}(t)|\hat{x}| s_{1}(t)\right\rangle+\left|c_{2}\right|^{2}\left\langle s_{2}(t)|\hat{x}| s_{2}(t)\right\rangle \\
= & \left|c_{1}\right|^{2} l+\left|c_{2}\right|^{2} T \pi \sqrt{8 E / m} .
\end{aligned}
$$

Proposition 3.2. (i) Suppose that a nucleus $n$ is in the superposition state $\left|\Psi_{t}\right\rangle_{n}\left(\left|\Psi_{t}\right\rangle_{n}\right.$-particle) given by Equation (3.19). Then the collaps: $\mid$ live cat $\rangle \rightarrow \mid$ death cat $\rangle$ arises at instant

$$
T_{\text {col }} \approx \frac{L \pm l}{\left|c_{2}\right|^{2} \sqrt{8 \pi^{2} E / m}}
$$

with a probability $\boldsymbol{P}_{T_{\text {col }}}(\mid$ death cat $\left.\rangle\right)$ to observe a state $\mid$ death cat $\rangle$ at instant $T_{\text {col }}$ is $\boldsymbol{P}_{T_{\text {col }}}(\mid$ death cat $\left.\rangle\right)=\left|c_{2}\right|^{2}$.

(ii) Assume now a Schrödinger's cat has performed the single measurement of $\left|\Psi_{t}\right\rangle_{n}$-particle position with accuracy of $\delta x=l$ at instant $T=T_{\text {col }}$ (given by Equation (3.29)) by internal monitor (see Figure 1) and the result $x \approx L \pm l$ is not observed by Schrödinger's cat. Then the collaps: |live cat $\rangle \rightarrow \mid$ death cat $\rangle$ never arises at any instant $T>T_{\text {col }}$ and a probability $\boldsymbol{P}_{T>T_{\text {col }}}(\mid$ death cat $\left.\rangle\right)$ to observe a state |death cat $\rangle$ at instant $T>T_{\text {col }}$ is $\boldsymbol{P}_{T>T_{\text {col }}}(\mid$ death cat $\left.\rangle\right)=0$.

Proof.(i) Note that for $t>0$ the marginal density matrix $\rho(t)$ is diagonal

$$
\rho(t)=\left(\begin{array}{cc}
\left|c_{1}\right|^{2}\left|\Psi_{\mathrm{II}}^{\#}(x, t)\right|^{2} \mathrm{~d} x & 0 \\
0 & \left|c_{2}\right|^{2}\left|\Psi_{\mathrm{I}}^{\#}(x, t)\right|^{2} \mathrm{~d} x
\end{array}\right) .
$$

In this case a Schrödinger's cat in fact performs the single measurement of $\left|\Psi_{t}\right\rangle_{n}$-particle position with accuracy of $\delta x=l$ at instant $t=T_{\text {col }}$ (given by Equation (3.29)) by internal monitor (see Figure 1). The probability of getting a result $L$ at instant $T \approx T_{\text {col }}$ with accuracy of $\delta x=l$ given by 


$$
\begin{aligned}
& \int_{|L-x| \leq I / 2}\left|\left\langle x \mid \Psi_{T}\right\rangle_{n}\right|^{2} \mathrm{~d} x \\
= & \left.\int_{|L-x| \leq I / 2}\left|\left\langle x\left|c_{1}\right| s_{1}(T)\right\rangle+c_{2}\right| s_{2}(T)\right\rangle\left.\right|^{2} \mathrm{~d} x \\
= & \int_{|L-x| \leq I / 2}\left|c_{1}\left\langle x \mid s_{1}(T)\right\rangle+c_{2}\left\langle x \mid S_{2}(T)\right\rangle\right|^{2} \mathrm{~d} x \\
= & \int_{|L-x| \leq I / 2}\left|c_{1}^{2} \Psi_{\text {II }}^{\# 2}(x, T)+c_{2}^{2} \Psi_{\mathrm{I}}^{\# 2}(x, T)+2 c_{1} C_{2} \Psi_{\mathrm{I}}^{\#}(x, T) \Psi_{\text {II }}^{\#}(x, T)\right| \mathrm{d} x .
\end{aligned}
$$

From Equation (3.30) by Remark 3.7 and Equation (3.13) one obtains

$$
\begin{aligned}
\int_{|L-x| \leq I / 2}\left|\left\langle x \mid \Psi_{T}\right\rangle_{n}\right|^{2} \mathrm{~d} x & =\int_{|L-x| \leq \leq 1 / 2}\left|c_{2}^{2} \Psi_{\mathbf{I}}^{\# 2}(x, T)\right| \mathrm{d} x \\
& =\left|c_{2}\right|^{2} \int_{|L-x| \leq I / 2}\left|\Psi_{\mathbf{I}}^{\#}(x, T)\right|^{2} \mathrm{~d} x=\left|c_{2}\right|^{2} .
\end{aligned}
$$

Note that. When Schrödinger's cat has performed this measurement and the result $x \approx L \pm l$ is observed, then the immediate post measurement state of $\alpha$ -particle (by von Neumann postulate C.4) will end up in the state

$$
\begin{aligned}
& \left|\Psi_{T_{\text {col }}}\right\rangle_{n} \\
& =\frac{\int_{|L-x| \leq I / 2}|x\rangle\left\langle x|| \Psi_{\left.T_{\text {col }}\right\rangle_{n}} \mathrm{~d} x\right.}{\sqrt{\int_{|L-x| \leq 1 / 2}\left|\left\langle x|| \Psi_{T_{\text {col }}}\right\rangle_{n}\right|^{2} \mathrm{~d} x}}=\frac{\int_{|L-x| \leq l / 2}|x\rangle\left\langlex \left|\left(c_{1}\left|s_{1}\left(T_{\text {col }}\right)\right\rangle+C_{2}\left|s_{2}\left(T_{\text {col }}\right)\right\rangle\right) \mathrm{d} x\right.\right.}{\sqrt{\int_{|L-x| \leq 1 / 2}\left|\left\langle x \mid \Psi_{T_{\text {col }}}\right\rangle_{n}\right|^{2} \mathrm{~d} x}} \\
& =\frac{c_{1} \int_{|L-x| \leq I / 2}|x\rangle\left\langle x\left|s_{1}\left(T_{\text {col }}\right)+c_{2} \int_{|L-x| \leq I / 2}\right| x\right\rangle\left\langle x \mid s_{2}\left(T_{\text {col }}\right)\right\rangle \mathrm{d} x}{\sqrt{\int_{|L-x| \leq I / 2}\left|\left\langle x \mid \Psi_{T_{\text {col }}}\right\rangle_{\boldsymbol{n}}\right|^{2} \mathrm{~d} x}} \in \boldsymbol{S}_{\Theta}, \\
& \Theta=\{x \mid L-x \leq l / 2\} .
\end{aligned}
$$

From Equation (3.32) by Equation (3.31) and by Remark 3.7 one obtains

$$
\begin{aligned}
\mid \Psi_{\left.T_{\text {col }}\right\rangle_{n}} & =\frac{\int_{|L-x| \leq l / 2}|x\rangle\left\langle x \mid \Psi_{T_{\text {col }}}\right\rangle_{n} \mathrm{~d} x}{\sqrt{\int_{|L-x| \leq l / 2}\left|\left\langle x \mid \Psi_{T_{\text {col }}}\right\rangle_{n}\right|^{2} \mathrm{~d} x}}=\frac{\int_{|L-x| \leq l / 2}|x\rangle\left\langlex \left|\left(c_{1}\left|S_{1}\left(T_{\text {col }}\right)\right\rangle+c_{2}\left|s_{2}\left(T_{\text {col }}\right)\right\rangle\right) \mathrm{d} x\right.\right.}{\sqrt{\int_{|L-x| \leq l / 2}\left|\left\langle x \mid \Psi_{T_{\text {col }}}\right\rangle_{\boldsymbol{n}}\right|^{2} \mathrm{~d} x}} \\
& =\frac{c_{2}}{\left|c_{2}\right|} \int_{|L-x| \leq l / 2}|x\rangle\left\langle x \mid s_{2}\left(T_{\text {col }}\right)\right\rangle \mathrm{d} x .
\end{aligned}
$$

Obviously by Remark 3.4 the state $\left|\Psi_{T_{\text {col }}}\right\rangle_{n}$ kills Schrödinger's cat with a probability $\boldsymbol{P}_{T_{\text {col }}}(\mid$ death cat $\left.\rangle\right)=1$.

Proof.(ii) The probability of getting a result $L$ at any instant $T>T_{\text {col }}$ with accuracy of $\delta x=l$ by Equation (3.31) and Equation (3.13) given by

$$
\begin{aligned}
\int_{|L-x| \leq l / 2}\left|\left\langle x \mid \Psi_{T}\right\rangle_{n}\right|^{2} \mathrm{~d} x & =\int_{|L-x| \leq l / 2}\left|c_{2}^{2} \Psi_{\mathbf{I}}^{\# 2}(x, T)\right| \mathrm{d} x=\left|c_{2}\right|^{2} \int_{|L-x| \leq l / 2}\left|\Psi_{\mathbf{I}}^{\#}(x, T)\right|^{2} \mathrm{~d} x \\
& \simeq\left(\pi r_{c}^{2}\right)^{-1 / 2} \int_{|L-x| \leq l / 2} \theta_{1}(x+\pi T \sqrt{8 E / m}, l) \exp \left[-\frac{(x+\pi T \sqrt{8 E / m})^{2}}{r_{c}^{2}}\right] \\
& \simeq 0 .
\end{aligned}
$$

Thus standard formalism of continuous quantum measurements [2] [3] [4] [5] leads to a definite but unpredictable measurement outcomes, either $\left|s_{1}(t)\right\rangle$ or $\left|s_{2}(t)\right\rangle$ and thus $\left|\Psi_{t}\right\rangle_{n}$ suddenly "collapses" at unpredictable instant $t^{\prime}$ into the corresponding state $\left|s_{i}\left(t^{\prime}\right)\right\rangle, i=1,2$. 


\subsection{Resolution of the Schrödinger's Cat Paradox Using Generalized Von Neumann Postulate}

Proposition 3.3. Suppose that a nucleus $\boldsymbol{n}$ is in the superposition state given by Equation (3.19). The collaps: $\mid$ live cat $\rangle \rightarrow \mid$ death cat $\rangle$ arises at instant

$$
T=\frac{L}{\left|c_{2}\right|^{2} \sqrt{8 \pi^{2} E / m}}
$$

with a probability $\boldsymbol{P}_{T}(\mid$ death cat $\left.\rangle\right)$ to observe a state $\mid$ death cat $\rangle$ at instant $T$ is $\boldsymbol{P}_{T}(\mid$ death cat $\left.\rangle\right)=1$.

Proof. Let us consider now a state $\left|\Psi_{t}\right\rangle_{n}$ given by Equation (3.19). This state consists of a sum of two wave packets $c_{1} \Psi_{\mathrm{II}}^{\#}(x, t)$ and $c_{2} \Psi_{\mathrm{I}}^{\#}(x, t)$. Wave packet $c_{1} \Psi_{\mathrm{II}}^{\#}(x, t)$ present an $\alpha_{\text {II }}$-particle which lives in region II with a probability $\left|c_{1}\right|^{2}$ (see Figure 2 ). Wave packet $c_{2} \Psi_{\mathrm{I}}^{\#}(x, t)$ present an $\alpha_{\mathrm{I}}$-particle which lives in region $\mathbf{I}$ with a probability $\left|c_{2}\right|^{2}$ (see Figure 2) and moves from the right to the left. Note that $\mathbf{I} \cap \mathbf{I I}=\varnothing$. From Equation (3.28) follows that $\alpha_{\mathrm{I}}$-particle at each instant $t \geq 0$ moves quasiclassically from right to left by the law

$$
x(t)=-\left|c_{2}\right|^{2} \pi t \sqrt{8 E / m} .
$$

From Equation (3.34) one obtains

$$
T=T_{\text {col }} \simeq \frac{L}{\left|c_{2}\right| \sqrt{8 \pi^{2} E / m}} .
$$

Note that, in this case Schrödinger's cat in fact performs a single measurement of $\left|\Psi_{t}\right\rangle_{n}$-particle position with accuracy of $\delta x=l$ at instant $t=T=T_{\text {col }}$ (given by Equation (3.35)) by internal monitor (see Figure 1). The probability of getting the result $L$ at instant $t=T_{\text {col }}$ with accuracy of $\delta x=l$ by Remark 3.7 and by postulate C.V.2 and by postulate C.IV.3 (see Appendix C) given by (for complete explanation and motivation see [16])

$$
\begin{aligned}
& \int_{|L-x| \leq 1 / 2}\left[\left|\left\langle x\left|c_{1}\right| s_{1}\left(T_{\text {col }}\right)\right\rangle\right|^{2} *\left|\left\langle x\left|c_{2}\right| s_{2}\left(T_{\text {col }}\right)\right\rangle\right|^{2}\right] \mathrm{d} x \\
& =\int_{|L-x| \leq l / 2}\left|c_{2}\right|^{-2}\left|c_{1}\right|^{-2}\left[\left|\left\langle x\left|c_{1}\right|^{-2}|| s_{1}\left(T_{\text {col }}\right)\right\rangle\right|^{2} *\left|\left\langle x\left|c_{2}\right|^{-2}|| s_{2}\left(T_{\text {col }}\right)\right\rangle\right|^{2}\right] \mathrm{d} x \\
& =\int_{|L-x| \leq l / 2}\left|c_{2}\right|^{-2}\left|c_{1}\right|^{-2}\left[\left|\Psi_{\mathrm{I}}^{\#}\left(x\left|c_{2}\right|^{-2}, T_{\text {col }}\right)\right|^{2} *\left|\Psi_{\text {II }}^{\#}\left(x\left|c_{1}\right|^{-2}, T_{\text {col }}\right)\right|^{2}\right] \mathrm{d} x \\
& =1 .
\end{aligned}
$$

Note that, when Schrödinger's cat has performed this measurement and the result $x \approx L \pm l$ is observed, then the immediate post measurement state of $\alpha$ -particle (by generalized Von Neumann postulate C.V.3) will end up in the state

$$
\begin{aligned}
\left|\Psi_{T_{\text {col }}}\right\rangle_{n} & =\frac{\int_{|L-x| \leq 1 / 2}|x\rangle\left\langle x \mid \Psi_{T_{\text {col }}}\right\rangle_{n} \mathrm{~d} x}{\sqrt{\int_{|L-x| \leq l / 2}\left[\left|\left\langle x \mid s_{1}\left(T_{\text {col }}\right)\right\rangle\right|^{2}+\left|\left\langle x \mid s_{2}\left(T_{\text {col }}\right)\right\rangle\right|^{2}\right] \mathrm{d} x}} \\
& =\frac{\int_{|L-x| \leq I / 2}|x\rangle\left\langle x \mid s_{2}^{c_{2}}\left(T_{\text {col }}\right)\right\rangle \mathrm{d} x}{\sqrt{\int_{|L-x| \leq l / 2}\left[\left|\left\langle x \mid s_{2}\left(T_{\text {col }}\right)\right\rangle\right|^{2}\right] \mathrm{d} x}} \in \boldsymbol{H}_{\Theta}, \Theta=\{x \mid L-x \leq l / 2\} .
\end{aligned}
$$


The state $\left|\Psi_{T_{\mathrm{col}}}\right\rangle_{n}$ again kills Schrödinger's cat with a probability $\boldsymbol{P}_{T_{\text {col }}}(\mid$ death cat $\left.\rangle\right)=1$.

Thus is the collapsed state of the cat always shows definite and predictable outcomes even if cat also consists of a superposition:

$$
\left.\left.\mid \text { cat }\rangle=c_{1} \mid \text { live cat }\right\rangle+c_{2} \mid \text { death cat }\right\rangle \text {. }
$$

Contrary to van Kampen's [10] and some others' opinions, "looking" at the outcome changes nothing, beyond informing the observer of what has already happened.

We remain: there are widespread claims that Schrödinger's cat is not in a definite alive or dead state but is, instead, in a superposition of the two. van Kampen, for example, writes "The whole system is in a superposition of two states: one in which no decay has occurred and one in which it has occurred. Hence, the state of the cat also consists of a superposition: $\mid$ cat $\rangle=c_{1} \mid$ live cat $\rangle+c_{2} \mid$ death cat $\rangle$. The state remains a superposition until an observer looks at the cat" [10].

\section{Conclusions}

A new quantum mechanical formalism based on the probability representation of quantum states is proposed (for complete explanation see [17]). This paper in particular deals with the special case of the measurement problem, known as Schrödinger's cat paradox. We pointed out that Schrödinger's cat demands to reconcile Born's rule. Using new quantum mechanical formalism we find that the collapsed state of the Schrödinger's cat always shows definite and predictable outcomes even if cat also consists of a superposition (see [16] [17] [18])

$$
\begin{aligned}
& \left.\left.\mid \text { cat }\rangle=c_{1} \mid \text { live cat }\right\rangle+c_{2} \mid \text { death cat }\right\rangle \\
& \left|c_{1}\right|^{2}+\left|c_{2}\right|^{2}=1 .
\end{aligned}
$$

Using new quantum mechanical formalism the EPRB-paradox is considered successfully. We find that the EPRB-paradox can be resolved by nonprincipal and convenient relaxing of the Einstein's locality principle.

\section{Acknowledgements}

We thank the Editor and the referee for their comments.

\section{References}

[1] Weinberg, S. (2012) Collapse of the State Vector. Physical Review A, 85, Article ID: 062116. https://doi.org/10.1103/PhysRevA.85.062116

[2] Bassi, A., Lochan, K., Satin, S., Singh, T.P. and Ulbricht, H. (2013) Models of Wave Function Collapse, Underlying Theories, and Experimental Tests. Reviews of Modern Physics, 85, 471-527. http://arxiv.org/abs/1204.4325 https://doi.org/10.1103/RevModPhys.85.471

[3] Jacobs, K. and Steck, D.A. (2006) A Straightforward Introduction to Continuous Quantum Measurement. Contemporary Physics, 47, 279. https://doi.org/10.1080/00107510601101934

[4] Mensky, M.B. (1993) Continuous Quantum Measurements and Path Integrals. CRC 
Press, Boca Raton, 188 p.

[5] Mensky, M.B. (2000) Quantum Measurements and Decoherence: Models and Phenomenology. Springer Science \& Business Media, Berlin, 231 p.

[6] Leggett, A.J. (1984) Schrödinger's Cat and Her Laboratory Cousins. Contemporary Physics, 25, 583-598. https://doi.org/10.1080/00107518408210731

[7] Hobson, A. (2013) Two-Photon Interferometry and Quantum State Collapse. Physical Review A, 88, Article ID: 022105.

[8] Gamow, G. (1928) Zur Quantentheorie des Atomkernes. Zeitschrift für Physik, 51, 204. https://doi.org/10.1007/BF01343196

[9] Feynman, R. and Hibbs, A. (2010) Quantum Mechanics and Path Integrals. Dover Emended Edition, Mineola.

[10] Van Kampen, N.G. (1988) Ten Theorems about Quantum Mechanical Measurements. Physica A, 153, 97-113.

[11] Schrödinger, E. (1935) Die gegenwärtige Situation in der Quantenmechanik (The Present Situation in Quantum Mechanics). Naturwissenschaften, 23, 807-812.

[12] Wimmel, H. (1992) Quantum Physics \& Observed Reality: A Critical Interpretation of Quantum Mechanics. World Scientific, Singapore, 2. https://doi.org/10.1142/1691

[13] Faye, J. (2002) Copenhagen Interpretation of Quantum Mechanics. Stanford Encyclopedia of Philosophy. The Metaphysics Research Lab Center for the Study of Language and Information, Stanford University, Stanford.

[14] Carpenter, R.H.S. and Anderson, A.J. (2006) The Death of Schroedinger's Cat and of Consciousness-Based Wave-Function Collapse. Annales de la Fondation Louis de Broglie, 31, 45-52.

[15] Omnès, R. (1999) Quantum Philosophy. Understanding and Interpreting Contemporary Science. English Edition, Princeton University Press, Princeton.

[16] Foukzon, J., Potapov, A.A., Menkova, E.R. and Podosenov, S.A. (2016) A New Quantum Mechanical Formalism Based on the Probability Representation of Quantum States. 179 p. http://vixra.org/abs/1612.0298

https://www.researchgate.net/publication/309398584_A_new_quantum_mechanica _lformalism_based_on_the_probability_representation_of_quantum_states

[17] Foukzon, J., Potapov, A.A. and Podosenov, S.A. (2011) Exact Quasiclassical Asymptotics beyond Maslov Canonical Operator. http://arxiv.org/abs/1110.0098v5

[18] Foukzon, J., Potapov, A.A. and Podosenov, S.A. (2014) Schrödinger's Cat Paradox Resolution Using GRW Collapse Model. International Journal of Recent Advances in Physics, 3, 17-30. http://wireilla.com/physics/ijrap/papers/3314ijrap02.pdf 


\section{Appendix A}

The time-dependent Schrodinger equation governs the time evolution of a quantum mechanical system:

$$
i \hbar \frac{\partial \Psi(\boldsymbol{x}, t)}{\partial t}=\hat{\boldsymbol{H}} \Psi(\boldsymbol{x}, t) .
$$

The average, or expectation, value $\left\langle x_{i}\right\rangle$ of an observable $x_{i}$ corresponding to a quantum mechanical operator $\hat{x}_{i}$ is given by:

$$
\left\langle x_{i}\right\rangle\left(t, x_{0}, t_{0} ; \hbar\right)=\frac{\int_{\mathbb{R}^{d}} x_{i}\left|\Psi\left(x, t, x_{0}, t_{0} ; \hbar\right)\right|^{2} \mathrm{~d}^{d} x}{\int_{\mathbb{R}^{d}}\left|\Psi\left(x, t, x_{0}, t_{0} ; \hbar\right)\right|^{2} \mathrm{~d}^{d} x}, \quad i=1, \cdots, d .
$$

Remark A.1. We assume now that: the solution $\Psi\left(x, t, x_{0}, t_{0} ; \hbar\right)$ of the timedependent Schrödinger Equation (A.1) has a good approximation by a delta function such that

$$
\begin{aligned}
& \left|\Psi\left(x, t, x_{0}, t_{0} ; \hbar\right)\right|^{2} \simeq \prod_{i=1}^{d} \delta\left(x_{i}-x_{i}\left(t, x_{0}, t_{0}\right)\right), \\
& x_{i}\left(t, x_{0}, t_{0}\right)=x_{i, 0}, \\
& i=1, \cdots, d .
\end{aligned}
$$

Remark A.2. Note that under conditions given by Equation (A.3) QM-system which governed by Schrödinger Equation (A.1) completely evolve quasiclassically i.e. estimating the position $\left\{x_{i}\left(t, x_{0}, t_{0} ; \hbar\right)\right\}_{i=1}^{d}$ at each instant $t$ with final error $\delta$ gives $\left|\left\langle x_{i}\right\rangle\left(t, x_{0}, t_{0} ; \hbar\right)-x_{i}\left(t, x_{0}, t_{0}\right)\right| \leq \delta, i=1, \cdots, d$ with a probability

$$
\boldsymbol{P}\left\{\left|\left\langle x_{i}\right\rangle\left(t, \boldsymbol{x}_{0}, t_{0} ; \hbar\right)-x_{i}\left(t, \boldsymbol{x}_{0}, t_{0}\right)\right| \leq \delta\right\} \simeq 1 \text {. }
$$

Thus from Equation (A.2) and Equation (A.3) we obtain

$$
\begin{aligned}
\left\langle x_{i}\right\rangle\left(t, x_{0}, t_{0} ; \hbar\right) \simeq \frac{\int_{\mathbb{R}^{d}} x_{i} \prod_{i=1}^{d=1} \delta\left(x_{i}-x_{i}\left(t, x_{0}, t_{0}\right)\right) \mathrm{d}^{d} x}{\int_{\mathbb{R}^{d}}^{d=1} \prod_{i=1}^{d} \delta\left(x_{i}-x_{i}\left(t, x_{0}, t_{0}\right)\right) \mathrm{d}^{d} x} \\
=x_{i}\left(t, x_{0}, t_{0}\right) . \\
i=1, \cdots, d .
\end{aligned}
$$

Thus under condition given by Equation (A.3) one obtains

$$
\begin{aligned}
& \left\langle x_{i, t}\right\rangle\left(t, x_{0}, t_{0} ; \hbar\right) \simeq x_{i}\left(t, x_{0}, t_{0}\right), \\
& i=1, \cdots, d .
\end{aligned}
$$

Remark A.3. Let $\Psi_{i}\left(x, t, x_{0}, t_{0}\right), i=1,2$ be the solutions of the time-dependent Schrödinger Equation (A.1). We assume now that $\Phi\left(\boldsymbol{x}, t, \boldsymbol{x}_{0}, \boldsymbol{y}_{0}, t_{0}\right)$ is a linear superposition such that

$$
\begin{aligned}
& \Phi\left(\boldsymbol{x}, t, \boldsymbol{x}_{0}, \boldsymbol{y}_{0}, t_{0}\right)=c_{1} \Psi_{1}\left(\boldsymbol{x}, t, \boldsymbol{x}_{0}, t_{0}\right)+c_{2} \Psi_{2}\left(\boldsymbol{x}, t, \boldsymbol{y}_{0}, t_{0}\right) . \\
& \left|c_{1}\right|^{2}+\left|c_{2}\right|^{2}=1 .
\end{aligned}
$$

Then we obtain 


$$
\begin{aligned}
\left|\Phi\left(\boldsymbol{x}, t, \boldsymbol{x}_{0}, \boldsymbol{y}_{0}, t_{0}\right)\right|^{2} \\
=\left(\Phi\left(\boldsymbol{x}, t, \boldsymbol{x}_{0}, \boldsymbol{y}_{0}, t_{0}\right) \Phi^{*}\left(\boldsymbol{x}, t, \boldsymbol{x}_{0}, \boldsymbol{y}_{0}, t_{0}\right)\right) \\
=\left(\left[c_{1} \Psi_{1}\left(\boldsymbol{x}, t, \boldsymbol{x}_{0}, t_{0}\right)+c_{2} \Psi_{2}\left(\boldsymbol{x}, t, \boldsymbol{y}_{0}, t_{0}\right)\right]\right) \\
\quad \times\left(\left[c_{1}^{*} \Psi_{1}^{*}\left(\boldsymbol{x}, t, \boldsymbol{x}_{0}, t_{0}\right)+c_{2}^{*} \Psi_{2}^{*}\left(\boldsymbol{x}, t, \boldsymbol{x}_{0}, \boldsymbol{y}_{0}, t_{0}\right)\right]\right) \\
=\left|c_{1}\right|^{2}\left(\left|\Psi_{1}\left(\boldsymbol{x}, t, \boldsymbol{x}_{0}, t_{0}\right)\right|^{2}\right)+c_{1}^{*} c_{2}\left(\Psi_{1}^{*}\left(\boldsymbol{x}, t, \boldsymbol{x}_{0}\right) \Psi_{2}\left(\boldsymbol{x}, t, \boldsymbol{y}_{0}, t_{0}\right)\right) \\
+\left|c_{2}\right|^{2}\left(\left|\Psi_{2}\left(\boldsymbol{x}, t, \boldsymbol{y}_{0}, t_{0}\right)\right|^{2}\right)+c_{1} c_{2}^{*}\left(\Psi_{1}\left(\boldsymbol{x}, t, \boldsymbol{x}_{0}\right) \Psi_{2}^{*}\left(\boldsymbol{x}, t, \boldsymbol{y}_{0}, t_{0}\right)\right) .
\end{aligned}
$$

Definition A.1. Let $\langle\boldsymbol{x}\rangle\left(t, \boldsymbol{x}_{0}, \boldsymbol{y}_{0}, t_{0}\right)$ be a vector-function

$$
\begin{gathered}
\langle\boldsymbol{x}\rangle\left(t, \boldsymbol{x}_{0}, \boldsymbol{y}_{0}, t_{0}\right):[0, T] \times \mathbb{R}^{d} \times \mathbb{R}^{d} \times[0, T] \rightarrow \mathbb{R}^{d} \\
\langle\boldsymbol{x}\rangle\left(t, \boldsymbol{x}_{0}, \boldsymbol{y}_{0}, t_{0}\right)=\left\{\left\langle x_{1}\right\rangle\left(t, \boldsymbol{x}_{0}, \boldsymbol{y}_{0}, t_{0}\right), \cdots,\left\langle x_{d}\right\rangle\left(t, \boldsymbol{x}_{0}, \boldsymbol{y}_{0}, t_{0}\right)\right\},
\end{gathered}
$$

where

$$
\begin{aligned}
& \left\langle x_{i}\right\rangle\left(t, \boldsymbol{x}_{0}, \boldsymbol{y}_{0}, t_{0}\right) \\
& =\int_{\mathbb{R}^{d}} x_{i}\left|\Phi\left(\boldsymbol{x}, t, \boldsymbol{x}_{0}, \boldsymbol{y}_{0}, t_{0}\right)\right|^{2} \mathrm{~d}^{d} x \\
& =\left|c_{1}\right|^{2} \int_{\mathbb{R}^{d}} x_{i}\left|\Psi_{1}\left(\boldsymbol{x}, t, \boldsymbol{x}_{0}, t_{0}\right)\right|^{2} \mathrm{~d}^{d} x+\left|c_{2}\right|^{2} \int_{\mathbb{R}^{d}} x_{i}\left|\Psi_{2}\left(\boldsymbol{x}, t, \boldsymbol{y}_{0}, t_{0}\right)\right|^{2} \mathrm{~d}^{d} x \\
& \quad+c_{1}^{*} C_{2} \int_{\mathbb{R}^{d}} x_{i} \Psi_{1}^{*}\left(\boldsymbol{x}, t, \boldsymbol{x}_{0}, t_{0}\right) \Psi_{2}\left(\boldsymbol{x}, t, \boldsymbol{y}_{0}, t_{0}\right) \mathrm{d}^{d} x \\
& \quad+c_{1} C_{2}^{*} \int_{\mathbb{R}^{d}} x_{i} \Psi_{1}\left(\boldsymbol{x}, t, \boldsymbol{x}_{0}, t_{0}\right) \Psi_{2}^{*}\left(\boldsymbol{x}, t, \boldsymbol{y}_{0}, t_{0}\right) \mathrm{d}^{d} x .
\end{aligned}
$$

Definition A.2. Let $\Delta\left(t, \boldsymbol{x}_{0}, \boldsymbol{y}_{0}, t_{0}\right)$ be a vector-function

$$
\begin{gathered}
\Delta\left(t, \boldsymbol{x}_{0}, \boldsymbol{y}_{0}, t_{0}\right):[0, T] \times \mathbb{R}^{d} \times \mathbb{R}^{d} \rightarrow \mathbb{R}^{d} \\
\left(\Delta\left(t, \boldsymbol{x}_{0}, \boldsymbol{y}_{0}, t_{0}\right)\right)=\left\{\delta_{1}\left(t, \boldsymbol{x}_{0}, \boldsymbol{y}_{0}, t_{0}\right), \cdots, \delta_{d}\left(t, \boldsymbol{x}_{0}, \boldsymbol{y}_{0}, t_{0}\right)\right\}
\end{gathered}
$$

where

$$
\begin{aligned}
\delta_{i}\left(t, \boldsymbol{x}_{0}, \boldsymbol{y}_{0}, t_{0}\right)= & \delta\left[x_{i}\left(t, \boldsymbol{x}_{0}, \boldsymbol{y}_{0}, t_{0}\right)\right] \\
= & c_{1}^{*} c_{2} \int_{\mathbb{R}^{d}} x_{i} \Psi_{1}^{*}\left(\boldsymbol{x}, t, \boldsymbol{x}_{0}, t_{0}\right) \Psi_{2}\left(\boldsymbol{x}, t, \boldsymbol{y}_{0}, t_{0}\right) \mathrm{d}^{d} x \\
& +c_{1} c_{2}^{*} \int_{\mathbb{R}^{d}} x_{i} \Psi_{1}\left(\boldsymbol{x}, t, \boldsymbol{x}_{0}, t_{0}\right) \Psi_{2}^{*}\left(\boldsymbol{x}, t, \boldsymbol{y}_{0}, t_{0}\right) \mathrm{d}^{d} x .
\end{aligned}
$$

Substituting Equation (A.11) into Equation (A.9) gives

$$
\begin{aligned}
& \left\langle x_{i}\right\rangle\left(t, \boldsymbol{x}_{0}, \boldsymbol{y}_{0}, t_{0}\right) \\
= & \int_{\mathbb{R}^{d}} x_{i}\left|\Phi\left(\boldsymbol{x}, t, \boldsymbol{x}_{0}, \boldsymbol{y}_{0}, t_{0}\right)\right|^{2} \mathrm{~d}^{d} x \\
= & \left|c_{1}\right|^{2} \int_{\mathbb{R}^{d}} x_{i}\left|\Psi_{1}\left(\boldsymbol{x}, t, \boldsymbol{x}_{0}, t_{0}\right)\right|^{2} \mathrm{~d}^{d} x \\
& +\left|c_{2}\right|^{2} \int_{\mathbb{R}^{d}} x_{i}\left|\Psi_{2}\left(\boldsymbol{x}, t, \boldsymbol{y}_{0}, t_{0}\right)\right|^{2} \mathrm{~d}^{d} x+\delta_{i}\left(t, \boldsymbol{x}_{0}, \boldsymbol{y}_{0}, t_{0}\right) \\
= & \left|c_{1}\right|^{2}\left\langle x_{i}\right\rangle\left(t, \boldsymbol{x}_{0}, t_{0}\right)+\left|c_{2}\right|^{2}\left\langle x_{i}\right\rangle\left(t, \boldsymbol{y}_{0}, t_{0}\right)+\delta_{i}\left(t, \boldsymbol{x}_{0}, \boldsymbol{y}_{0}, t_{0}\right) .
\end{aligned}
$$

Substitution Equation (A.5) into Equation (A.12) gives 


$$
\begin{aligned}
& \left\langle x_{i}\right\rangle\left(t, \boldsymbol{x}_{0}, \boldsymbol{y}_{0}, t_{0}\right) \\
= & \int_{\mathbb{R}^{d}} x_{i}\left|\Phi\left(\boldsymbol{x}, t, \boldsymbol{x}_{0}, \boldsymbol{y}_{0}, t_{0}\right)\right|^{2} \mathrm{~d}^{d} x \\
= & \left|c_{1}\right|^{2}\left\langle x_{i}\right\rangle\left(t, \boldsymbol{x}_{0}, t_{0}\right)+\left|c_{2}\right|^{2}\left\langle x_{i}\right\rangle\left(t, \boldsymbol{y}_{0}, t_{0}\right)+\delta_{i}\left(t, \boldsymbol{x}_{0}, \boldsymbol{y}_{0}, t_{0}\right) \\
\simeq & \left|c_{1}\right|^{2} x_{i}\left(t, \boldsymbol{x}_{0}, t_{0}\right)+\left|c_{2}\right|^{2} x_{i}\left(t, \boldsymbol{y}_{0}, t_{0}\right)+\delta_{i}\left(t, \boldsymbol{x}_{0}, \boldsymbol{y}_{0}, t_{0}\right) .
\end{aligned}
$$

\section{Appendix B}

The Schrödinger Equation (2.1) in region $\mathbf{I}=\{x \mid x<0\}$ has the following form

$$
\hbar^{2} \frac{\partial^{2} \Psi_{\mathbf{I}}(x)}{\partial x^{2}}+2 m E \Psi_{\mathbf{I}}(x)=0 \text {. }
$$

From Schrödinger Equation (B.1) it follows

$$
\hbar^{2} \int_{-\infty}^{0} \frac{\partial^{2} \Psi_{\mathbf{I}}(x)}{\partial x^{2}} \mathrm{~d} x+2 m E \int_{-\infty}^{0} \Psi_{\mathbf{I}}(x) \mathrm{d} x=0 .
$$

Let $\Psi_{\mathrm{I}}^{\#}(x)$ be a function

$$
\Psi_{\mathrm{I}}^{\#}(x)=\phi(x) \Psi_{\mathbf{I}}(x),
$$

where

$$
\phi(x)=\left(\pi r_{c}^{2}\right)^{-1 / 4} \exp \left(\frac{x^{2}}{2 r_{c}^{2}}\right)
$$

see Equation (2.9). Note that

$$
\begin{aligned}
& \frac{\partial^{2}\left[\phi(x) \Psi_{\mathbf{I}}(x)\right]}{\partial x^{2}} \\
= & \frac{\partial}{\partial x}\left[\Psi_{\mathbf{I}}(x) \frac{\partial \phi(x)}{\partial x}+\phi(x) \frac{\partial \Psi_{\mathbf{I}}(x)}{\partial x}\right] \\
= & 2 \frac{\partial \Psi_{\mathbf{I}}(x)}{\partial x} \frac{\partial \phi(x)}{\partial x}+\Psi_{\mathbf{I}}(x) \frac{\partial^{2} \phi(x)}{\partial x^{2}}+\phi(x) \frac{\partial^{2} \Psi_{\mathbf{I}}(x)}{\partial x^{2}} .
\end{aligned}
$$

Therefore substitution (B.2) into LHS of the Schrödinger Equation (B.1) gives

$$
\begin{aligned}
& \hbar^{2} \int_{-\infty}^{0} \frac{\partial^{2} \Psi_{\mathbf{I}}^{\#}(x)}{\partial x^{2}} \mathrm{~d} x+2 m E \int_{-\infty}^{0} \Psi_{\mathbf{I}}^{\#}(x) \mathrm{d} x \\
& =\hbar^{2} \int_{-\infty}^{0} \frac{\partial^{2} \phi(x) \Psi_{\mathbf{I}}(x)}{\partial x^{2}} \mathrm{~d} x+2 E m \int_{-\infty}^{0} \phi(x) \Psi_{\mathbf{I}}(x) \mathrm{d} x \\
& =2 \hbar^{2} \int_{-\infty}^{0} \frac{\partial \Psi_{\mathbf{I}}(x)}{\partial x} \frac{\partial \phi(x)}{\partial x} \mathrm{~d} x+\hbar^{2} \int_{-\infty}^{0} \Psi_{\mathbf{I}}(x) \frac{\partial^{2} \phi(x)}{\partial x^{2}} \mathrm{~d} x \\
& +\int_{-\infty}^{0} \phi(x)\left\{\hbar^{2} \frac{\partial^{2} \Psi_{\mathbf{I}}(x)}{\partial x^{2}}+2 E m \int_{-\infty}^{0} \Psi_{\mathbf{I}}(x)\right\} \mathrm{d} x .
\end{aligned}
$$

Note that

$$
\int_{-\infty}^{0} \phi(x)\left\{\hbar^{2} \frac{\partial^{2} \Psi_{\mathbf{I}}(x)}{\partial x^{2}}+2 E m \int_{-\infty}^{0} \Psi_{\mathbf{I}}(x)\right\} \mathrm{d} x=0 .
$$

Therefore from Equation (B.6) and Equations ((2.3) and (2.4)) one obtains 


$$
\begin{aligned}
& \hbar^{2} \int_{-\infty}^{0} \frac{\partial^{2} \Psi_{\mathbf{I}}^{\#}(x)}{\partial x^{2}} \mathrm{~d} x+2 m E \int_{-\infty}^{0} \Psi_{\mathbf{I}}^{\#}(x) \mathrm{d} x \\
& =\hbar^{2} \int_{-\infty}^{0} \frac{\partial^{2} \phi(x) \Psi_{\mathbf{I}}(x)}{\partial x^{2}} \mathrm{~d} x+2 E m \int_{-\infty}^{0} \phi(x) \Psi_{\mathbf{I}}(x) \mathrm{d} x \\
& =2 \hbar^{2} \int_{1}^{\infty} \frac{\partial \Psi_{\mathbf{I}}(x)}{\partial x} \frac{\partial \phi(x)}{\partial x} \mathrm{~d} x+\hbar^{2} \int_{1}^{\infty} \Psi_{\mathbf{I}}(x) \frac{\partial^{2} \phi(x)}{\partial x^{2}} \mathrm{~d} x .
\end{aligned}
$$

From Equation (B.6) one obtains

$$
\begin{aligned}
& \frac{\partial \phi(x)}{\partial x}=\left(\pi r_{c}^{2}\right)^{-1 / 4} \frac{\partial}{\partial x} \exp \left[-\frac{x^{2}}{2 r_{c}^{2}}\right]=-\left(\pi r_{c}^{2}\right)^{-1 / 4} r_{c}^{-2} x \exp \left[-\frac{x^{2}}{2 r_{c}^{2}}\right], \\
& \frac{\partial^{2} \phi(x)}{\partial x^{2}}=-\left(\pi r_{c}^{2}\right)^{-1 / 4} r_{c}^{-2} \exp \left[-\frac{x^{2}}{2 r_{c}^{2}}\right]+\left(\pi r_{c}^{2}\right)^{-1 / 4} r_{c}^{-4} x^{2} \exp \left[-\frac{x^{2}}{2 r_{c}^{2}}\right] .
\end{aligned}
$$

From Equation (B.9) and Equations ((2.3), (2.4)) one obtains

$$
\begin{aligned}
\hbar^{2} \int_{-\infty}^{0} \frac{\partial \Psi_{\mathbf{I}}(x)}{\partial x} \frac{\partial \phi(x)}{\partial x} \mathrm{~d} x & =-\frac{\hbar^{2}}{\left(\pi r_{c}^{2}\right)^{1 / 4} r_{c}^{2}} \int_{-\infty}^{0} \frac{\partial \exp (i k x)}{\partial x} x \exp \left[-\frac{x^{2}}{2 r_{c}^{2}}\right] \mathrm{d} x \\
& =-\frac{2 \pi \sqrt{2 m E} \hbar}{\left(\pi r_{c}^{2}\right)^{1 / 4} r_{c}^{2}} \int_{-\infty}^{0} x \exp \left(i \frac{2 \pi \sqrt{2 m E}}{\hbar} x\right) \exp \left[-\frac{x^{2}}{2 r_{c}^{2}}\right] \mathrm{d} x, \\
k & =\frac{2 \pi}{\hbar} \sqrt{2 m E}
\end{aligned}
$$

and

$$
\begin{aligned}
\hbar^{2} \int_{-\infty}^{0} \Psi_{\mathbf{I}}(x) \frac{\partial^{2} \phi(x)}{\partial x^{2}} \mathrm{~d} x= & -\frac{\hbar^{2}}{\left(\pi r_{c}^{2}\right)^{3 / 4} r_{c}^{2}} \int_{-\infty}^{0} \exp (i k x) \exp \left[-\frac{x^{2}}{2 r_{c}^{2}}\right] \mathrm{d} x \\
& +\frac{\hbar^{2}}{\left(\pi r_{c}^{2}\right)^{1 / 4} r_{c}^{2}} \int_{-\infty}^{0} x^{2} \exp (i k x) \exp \left[-\frac{x^{2}}{2 r_{c}^{2}}\right] \mathrm{d} x .
\end{aligned}
$$

\section{Appendix C. Generalized Postulates for Continuous Valued Observables}

Suppose we have an $\mathrm{n}$-dimensional physical quantum system with continuous observables.

\section{Then we claim the following:}

C.I. Any given $n$-dimensional quantum system is identified by a set $\boldsymbol{Q}^{s}$ : $\left.\boldsymbol{Q}^{s} \triangleq\left\langle\boldsymbol{H}, \mathfrak{I}, \mathfrak{R}, £_{2,1}, \mathfrak{J}^{*}(\boldsymbol{H}), \boldsymbol{G}, \mid \psi_{t}\right\rangle\right\rangle$

where:

(i) $\boldsymbol{H}$ that is some infinite-dimensional complex Hilbert space,

(ii) $\mathfrak{I}=(\Omega, \boldsymbol{P})$ that is complete probability space,

(iii) $\mathfrak{R}=\left(\mathbb{R}^{n}, \Sigma\right)$ that is measurable space,

(iv) $£_{2,1}(\Omega)$ that is complete space of random variables $X: \Omega \rightarrow \mathbb{R}^{n}$ such that $\int_{\Omega}\|X(\omega)\| \mathrm{d} \boldsymbol{P}<\infty, \int_{\Omega}\|X(\omega)\|^{2} \mathrm{~d} \boldsymbol{P}<\infty$

(v) $\boldsymbol{G}: \mathfrak{I}^{*}(\boldsymbol{H}) \times \boldsymbol{H} \rightarrow £_{2,1}(\Omega)$ that is one to one correspondence such that

$$
\begin{aligned}
& |\langle\psi|\hat{Q}| \psi\rangle|=\int_{\Omega}(\boldsymbol{G}[\hat{Q},|\psi\rangle](\omega)) \mathrm{d} \boldsymbol{P}=\boldsymbol{E}_{\Omega}(\boldsymbol{G}[\hat{Q},|\psi\rangle](\omega)), \\
& \boldsymbol{G}[\hat{\mathbf{1}},|\psi\rangle](\omega)=1
\end{aligned}
$$


for any $|\psi\rangle \in \boldsymbol{H}$ and for any Hermitian operator $\hat{Q}: \boldsymbol{H} \rightarrow \boldsymbol{H}, \hat{Q} \in \mathfrak{I}^{*}(\boldsymbol{H}) \subset C^{*}(\boldsymbol{H})$, where $C^{*}(\boldsymbol{H})$ is $C^{*}$-algebra of the Hermitian adjoint operators in $\boldsymbol{H}$ and $\mathcal{I}^{*}(\boldsymbol{H})$ an commutative subalgebra of $C^{*}(\boldsymbol{H})$.

(vi) $\left|\psi_{t}\right\rangle$ is an continuous vector function $\left|\psi_{t}\right\rangle: \mathbb{R}_{+} \rightarrow \boldsymbol{H}$ which represented the evolution of the quantum system $\mathfrak{R}$.

C.I.2. For any $\left|\psi_{1}\right\rangle,\left|\psi_{2}\right\rangle \in \boldsymbol{H}$ and for any Hermitian operator $\hat{Q}: \boldsymbol{H} \rightarrow \boldsymbol{H}$ such that $\left\langle\psi_{1}|\hat{Q}| \psi_{2}\right\rangle=\left\langle\psi_{2}|\hat{Q}| \psi_{1}\right\rangle=0$ :

$$
\boldsymbol{G}\left[\hat{Q},\left|\psi_{1}\right\rangle+\left|\psi_{2}\right\rangle\right](\omega)=\boldsymbol{G}\left[\hat{Q},\left|\psi_{1}\right\rangle\right](\omega)+\boldsymbol{G}\left[\hat{Q},\left|\psi_{2}\right\rangle\right](\omega) .
$$

C.I.3. Suppose that the evolution of the quantum system is represented by continuous vector function $\left|\psi_{t}\right\rangle: \mathbb{R}_{+} \rightarrow \boldsymbol{H}$. Then any process of continuous measurements on measuring observable $\hat{Q}$ for the system in state $\left|\psi_{t}\right\rangle$ one can to describe by an continuous $\mathbb{R}^{n}$-valued stochastic processes $X_{t}(\omega)=X_{t}\left(\omega ; \hat{Q},\left|\psi_{t}\right\rangle\right)$ given on probability space $(\Omega, \mathcal{F}, \boldsymbol{P})$ and a measurable space $\left(\mathbb{R}^{n}, \Sigma\right)$.

Remark C.1. We assume now for short but without loss of generality that $n=1$.

Remark C.2. Let $X(\omega)$ be random variable $X(\omega) \in £_{2,1}(\Omega)$ such that $X(\omega)=\boldsymbol{G}[\hat{Q},|\psi\rangle](\omega)$, then we denote such random variable by $X_{\hat{Q}|\psi\rangle}(\omega)$ or simply $X_{|\psi\rangle}(\omega)$ for short. The probability density of random variable $X_{\hat{Q}|\psi\rangle}(\omega)$ we denote by $p_{\hat{Q}|\psi\rangle}(q), q \in \mathbb{R}$ or simply $p_{|\psi\rangle}(q)$ for short.

Definition C.1. The classical pure states correspond to vectors $\boldsymbol{v} \in \boldsymbol{H}$ of norm $\|v\| \equiv 1$. Thus the set of all classical pure states corresponds to the unit sphere $\boldsymbol{S}^{\infty} \subset \boldsymbol{H}$ in a Hilbert space $\boldsymbol{H}$.

Definition C.2. The projective Hilbert space $P(\boldsymbol{H})$ of a complex Hilbert space $\boldsymbol{H}$ is the set of equivalence classes $[\boldsymbol{v}]$ of vectors $\boldsymbol{v}$ in $\boldsymbol{H}$, with $\boldsymbol{v} \neq \mathbf{0}$, for the equivalence relation given by $\boldsymbol{v} \sim_{P} \boldsymbol{w} \Leftarrow \boldsymbol{v}=\lambda \boldsymbol{w}$ for some non-zero complex number $\lambda \in \mathbb{C}$. The equivalence classes for the relation $\sim_{P}$ are also called rays or projective rays.

Remark C.3. The physical significance of the projective Hilbert space $P(\boldsymbol{H})$ is that in canonical quantum theory, the states $|\psi\rangle$ and $\lambda|\psi\rangle$ represent the same physical state of the quantum system, for any $\lambda \neq 0$. It is conventional to choose a state $|\psi\rangle$ from the ray $[|\psi\rangle]$ so that it has unit norm $\sqrt{\langle\psi \mid \psi\rangle}=1$.

Remark C.4. In contrast with canonical quantum theory we have used instead contrary to $\sim_{P}$ equivalence relation $\sim_{Q}$, a Hilbert space $\boldsymbol{H}$ (see Definition C.7).

Definition C.3. The non-classical pure states correspond to the vectors $\boldsymbol{v} \in \boldsymbol{H}$ of a norm $\|v\| \neq 1$. Thus the set of all non-classical pure states corresponds to the set $\boldsymbol{H} \backslash \boldsymbol{S}^{\infty} \subset \boldsymbol{H}$ in the Hilbert space $\boldsymbol{H}$.

Suppose we have an observable $Q$ of a quantum system that is found through an exhaustive series of measurements, to have a set $\mathfrak{I}$ of values $q \in \mathfrak{I}$ such that $\mathfrak{I}=\cup_{i=1}^{m}\left(\theta_{1}^{i}, \theta_{2}^{i}\right), m \geq 2, \quad\left(\theta_{1}^{i}, \theta_{2}^{i}\right) \cap\left(\theta_{1}^{j}, \theta_{2}^{j}\right)=\varnothing, i \neq j$. Note that in practice any observable $Q$ is measured to an accuracy $\delta q$ determined 
by the measuring device. We represent now by $|q\rangle$ the idealized state of the system in the limit $\delta q \rightarrow 0$, for which the observable definitely has the value $q$.

\section{Then we claim the following:}

C.II.1. The states $\{|q\rangle: q \in \mathfrak{I}\}$ form a complete set of $\delta$-function normalized basis states for the state space $\boldsymbol{H}_{\mathfrak{J}}$ of the system.

That the states $\{|q\rangle: q \in \mathfrak{I}\}$ form a complete set of basis states means that any state $|\psi[\mathfrak{I}]\rangle \in \boldsymbol{H}_{\mathfrak{I}}$ of the system can be expressed as: $|\psi[\mathfrak{I}]\rangle=\int_{\mathfrak{I}} c_{\psi[\mathfrak{I}]}(q) \mathrm{d} q$, where $\operatorname{supp}\left(c_{\psi[\mathfrak{s}]}(q)\right) \subseteq \mathfrak{I}$ and while $\delta$-function normalized means that $\left\langle q \mid q^{\prime}\right\rangle=\delta\left(q-q^{\prime}\right)$ from which follows $c_{\psi[\Im]}(q)=\langle q \mid \psi[\Im]\rangle$ so that $|\psi[\mathfrak{I}]\rangle=\int_{\mathfrak{I}}|q\rangle\langle q \mid \psi[\mathfrak{I}]\rangle \mathrm{d} q$.

The completeness condition can then be written as $\int_{\mathfrak{I}}|q\rangle\langle q| \mathrm{d} q=\hat{\mathbf{1}}_{H_{\Im}}$.

C.II.2. For the system in state $|\psi[\mathfrak{I}]\rangle$ the probability $P(q, q+\mathrm{d} q ;|\psi[\mathfrak{J}]\rangle)$ of obtaining the result $q \in \mathfrak{I}$ lying in the range $(q, q+\mathrm{d} q) \subset \mathfrak{I}$ on measuring observable $\hat{Q}$ is given by

$$
P(q, q+\mathrm{d} q ;|\psi[\mathfrak{I}]\rangle)=p_{|\psi[\mathfrak{I}]\rangle}(q) \mathrm{d} q
$$

for any $|\psi[\mathfrak{I}]\rangle \in \boldsymbol{H}_{\mathfrak{I}}$.

Remark C.5. Note that in general case $p_{|\psi[\Im]\rangle}(q) \neq\left|c_{\psi[\Im]}(q)\right|^{2}$.

C.II.3. The observable $Q_{\mathfrak{I}}$ is represented by a Hermitian operator $\hat{Q}_{\mathfrak{I}}: \boldsymbol{H}_{\mathfrak{I}} \rightarrow \boldsymbol{H}_{\mathfrak{I}}$ whose eigenvalues are the possible results $\{q: q \in \mathfrak{I}\}$, of a measurement of $Q_{\mathfrak{I}}$, and the associated eigenstates are the states $\{|q\rangle: q \in \mathfrak{I}\}$, i.e. $\hat{Q}_{\mathfrak{J}}|q\rangle=q|q\rangle, q \in \mathfrak{I}$.

Remark C.6. Note that the spectral decomposition of the operator $\hat{Q}_{\mathfrak{I}}$ is then

$$
\hat{Q}_{\mathfrak{I}}=\int_{\mathfrak{S}} q|q\rangle\langle q| \mathrm{d} q .
$$

Definition C.4. A connected set in $\mathbb{R}$ is a set $X \subset \mathbb{R}$ that cannot be partitioned into two nonempty subsets which are open in the relative topology induced on the set. Equivalently, it is a set which cannot be partitioned into two nonempty subsets such that each subset has no points in common with the set closure of the other.

Definition C.5. The well localized pure states $|\psi[\Theta]\rangle$ with a support $\Theta=\left(\theta_{1}, \theta_{2}\right)$ correspond to vectors of norm 1 and such that: $\operatorname{supp}\left(c_{\psi[\Theta]}(q)\right)=\Theta$ is a connected set in $\mathbb{R}$ Thus the set of all well localized pure states corresponds to the unit sphere $\boldsymbol{S}_{\Theta}^{\infty} \varsubsetneqq \boldsymbol{S}^{\infty} \subset \boldsymbol{H}$ in the Hilbert space $\boldsymbol{H}_{\Theta} \varsubsetneqq \boldsymbol{H}$.

Suppose we have an observable $Q_{\Theta}$ of a system that is found through an exhaustive series of measurements, to have a continuous range of values $q: \theta_{1}<q<\theta_{2}$.

III. Then we claim the following:

C.III.1. For the system in well localized pure state $|\psi[\Theta]\rangle$ such that:

(i) $|\psi[\Theta]\rangle \in \boldsymbol{S}_{\Theta}^{\infty}$ and

(ii) $\operatorname{supp}\left(c_{\psi[\Theta]}(q)\right) \triangleq\left\{q \mid c_{\psi[\Theta]}(q) \neq 0\right\}$ is a connected set in $\mathbb{R}$, then the 
probability $P(q, q+\mathrm{d} q ;|\psi[\Theta]\rangle)$ of obtaining the result $q$ lying in the range $(q, q+\mathrm{d} q)$ on measuring observable $Q_{\Theta}$ is given by

$$
P(q, q+\mathrm{d} q ;|\psi[\Theta]\rangle)=|\langle q \mid \psi[\Theta]\rangle|^{2} \mathrm{~d} q=\left|c_{\psi[\Theta]}(q)\right|^{2} \mathrm{~d} q .
$$

C.III.2. $\quad p_{|\psi[\Theta]\rangle}(q) \mathrm{d} q=|\langle q \mid \psi[\Theta]\rangle|^{2} \mathrm{~d} q=\left|c_{\psi[\Theta]}(q)\right|^{2} \mathrm{~d} q$.

C.III.3. Let $\left|\psi\left[\Theta_{1}\right]\right\rangle$ and $\left|\psi\left[\Theta_{2}\right]\right\rangle$ be well localized pure states with $\Theta_{1}=\left(\theta_{1}^{1}, \theta_{2}^{1}\right)$ and $\Theta_{2}=\left(\theta_{1}^{2}, \theta_{2}^{2}\right)$ correspondingly. Let $X_{1}(\omega)=X_{\left|\psi\left[\Theta_{1}\right]\right\rangle}(\omega)$ and $X_{2}(\omega)=X_{\left|\psi\left[\Theta_{2}\right]\right\rangle}(\omega)$ correspondingly. Assume that $\bar{\Theta}_{1} \cap \bar{\Theta}_{2}=\varnothing$ (here the closure of $\Theta_{i}, i=1,2$ is denoted by $\left.\bar{\Theta}_{i}, i=1,2\right)$ then random variables $X_{1}(\omega)$ and $X_{2}(\omega)$ are independent.

C.III.4. If the system is in well localized pure state $|\psi[\Theta]\rangle$ the state $|\psi[\Theta]\rangle$ described by a wave function $\psi(q, \Theta)=\langle q \mid \psi[\Theta]\rangle$ and the value of observable $Q_{\Theta}$ is measured once each on many identically prepared system, the average value of all the measurements will be

$$
\left\langle Q_{\Theta}\right\rangle=\frac{\int_{\Theta} q|\psi(q, \Theta)|^{2} \mathrm{~d} q}{\int_{\Theta}|\psi(q, \Theta)|^{2} \mathrm{~d} q} .
$$

The completeness condition can then be written as $\int_{\Theta}|q\rangle\langle q| \mathrm{d} q=\hat{\mathbf{1}}_{\boldsymbol{H}_{\Theta}}$. Completeness means that for any state $|\psi[\Theta]\rangle \in \boldsymbol{S}_{\Theta}^{\infty}$ it must be the case that $\int_{\Theta}|\langle q \mid \psi[\Theta]\rangle|^{2} \mathrm{~d} q \neq 0$, i.e. there must be a non-zero probability to get some result on measuring observable $Q_{\Theta}$.

C.III.5. (von Neumann measurement postulate) Assume that

(i) $|\psi\rangle \in \boldsymbol{S}_{\Theta}^{\infty}$ and (ii) $\operatorname{supp}\left(c_{\psi}(q)\right)=\Theta$ is a connected set in $\mathbb{R}$. Then if on performing a measurement of $Q_{\Theta}$ with an accuracy $\delta q$, the result is obtained in the range $\left(q-\frac{1}{2} \delta q, q+\frac{1}{2} \delta q\right)$, then the system will end up in the state

$$
\frac{\hat{P}(q, \delta q)|\psi[\Theta]\rangle}{\sqrt{\langle\psi|\hat{P}(q, \delta q)| \psi[\Theta]\rangle}}=\frac{\int_{\left|q-q^{\prime}\right| \leq \delta q / 2}\left|q^{\prime}\right\rangle\left\langle q^{\prime} \mid \psi[\Theta]\right\rangle \mathrm{d} q^{\prime}}{\sqrt{\int_{\left|q-q^{\prime}\right| \leq \delta q / 2}\left|\left\langle q^{\prime} \mid \psi[\Theta]\right\rangle\right|^{2} \mathrm{~d} q^{\prime}}} .
$$

\section{We claim the following:}

C.IV.1 For the system in state $\left|\psi^{a}[\Theta]\right\rangle=a|\psi[\Theta]\rangle \in \boldsymbol{H}_{\Theta}$, where: (i) $|\psi[\Theta]\rangle \in \boldsymbol{S}_{\Theta}^{\infty},|a| \neq 1$,

(ii) $\operatorname{supp}\left(c_{\psi[\Theta]}(q)\right)$ is a connected set in $\mathbb{R}$ and (iii) $|\psi[\Theta]\rangle=\int_{\theta_{1}}^{\theta_{2}} c_{\psi[\Theta]}(q)|q\rangle \mathrm{d} q$

$$
\boldsymbol{G}\left[\hat{Q}_{\Theta},\left|\psi^{a}[\Theta]\right\rangle\right]=|a|^{2} \boldsymbol{G}\left[\hat{Q}_{\Theta},|\psi[\Theta]\rangle\right]
$$

C.IV.2. Assume that the system in state $\left|\psi^{a}[\Theta]\right\rangle=a|\psi[\Theta]\rangle \in \boldsymbol{H}_{\Theta}$, where (i) $|\psi[\Theta]\rangle \in S_{\Theta}^{\infty}, \quad|a| \neq 1$, (ii) $\operatorname{supp}\left(c_{\psi[\Theta]}(q)\right)$ is a connected set in $\mathbb{R}$ and (iii) $|\psi[\Theta]\rangle=\int_{\theta_{1}}^{\theta_{2}} c_{\psi[\Theta]}(q)|q\rangle \mathrm{d} q$.

Then if the system is in state $\left|\psi^{a}[\Theta]\right\rangle$ described by a wave function 
$\psi^{a}(q ; \Theta)=\left\langle q \| \psi^{a}[\Theta]\right\rangle$ and the value of observable $Q_{\Theta}$ is measured once each on many identically prepared system, the average value of all the measurements will be

$$
\left\langle Q_{\Theta}\right\rangle=\int_{\Theta} q\left|\psi^{a}(q ; \Theta)\right|^{2} \mathrm{~d} q
$$

C.IV.3. The probability $P\left(q, q+d q ;\left|\psi^{a}[\Theta]\right\rangle\right) \mathrm{d} q$ of obtaining the result $q$ lying in the range $(q, q+\mathrm{d} q)$ on measuring $Q_{\Theta}$ is

$$
P\left(q, q+\mathrm{d} q ;\left|\psi^{a}[\Theta]\right\rangle\right) \mathrm{d} q=|a|^{-2}\left|c_{\psi[\Theta]}\left(q|a|^{-2}\right)\right|^{2} \mathrm{~d} q .
$$

Remark C.7. Note that C.IV.3 immediately follows from C.IV.1 and C.III.2.

C.IV.4. (Generalized von Neumann measurement postulate) If on performing a measurement of observable $Q_{\Theta}$ with an accuracy $\delta q$, the result is obtained in the range $\left(q-\frac{1}{2} \delta q, q+\frac{1}{2} \delta q\right)$, then the system immediately after measurement will end up in the state

$$
\begin{aligned}
\frac{\hat{P}(q, \delta q)\left|\psi^{a}[\Theta]\right\rangle}{\sqrt{\langle\psi|\hat{P}(q, \delta q)| \psi[\Theta]\rangle}} & =\frac{\int_{\left|q-q^{\prime}\right| \leq \delta q / 2}\left|q^{\prime}\right\rangle\left\langle q^{\prime} \mid \psi^{a}[\Theta]\right\rangle \mathrm{d} q^{\prime}}{\sqrt{\int_{\left|q-q^{\prime}\right| \leq \delta q / 2}\left|\left\langle q^{\prime} \mid \psi[\Theta]\right\rangle\right|^{2} \mathrm{~d} q^{\prime}}} \\
& =\frac{a \int_{\left|q-q^{\prime}\right| \leq \delta q / 2}\left|q^{\prime}\right\rangle\left\langle q^{\prime} \mid \psi[\Theta]\right\rangle \mathrm{d} q^{\prime}}{\sqrt{\int_{\left|q-q^{\prime}\right| \leq \delta q / 2}\left|\left\langle q^{\prime} \mid \psi[\Theta]\right\rangle\right|^{2} \mathrm{~d} q^{\prime}}} \in \boldsymbol{H}_{\Theta} .
\end{aligned}
$$

C.V.1. Let $\left|\Psi^{a_{1}, a_{2}}\left[\Theta_{1}, \Theta_{2}\right]\right\rangle=\left|\psi_{1}^{a_{1}}\left[\Theta_{1}\right]\right\rangle+\left|\psi_{2}^{a_{2}}\left[\Theta_{2}\right]\right\rangle \in \boldsymbol{H}_{1,2} \triangleq \boldsymbol{H}_{\Theta_{1}} \oplus \boldsymbol{H}_{\Theta_{2}} \varsubsetneqq \boldsymbol{H}$, where

(i) $\left|\psi_{i}^{a_{i}}\left[\Theta_{i}\right]\right\rangle=a_{i}\left|\psi_{i}\left[\Theta_{i}\right]\right\rangle \in \boldsymbol{H}_{\Theta_{i}},\left|\psi_{i}\right\rangle=\left|\psi_{i}\left[\Theta_{i}\right]\right\rangle \in \boldsymbol{S}_{\Theta_{i}}^{\infty},\left|a_{i}\right| \neq 1, i=1,2$;

(ii) $\operatorname{supp}\left(c_{\psi_{i}\left[\Theta_{i}\right]}(q)\right), i=1,2$ is a connected sets in $\mathbb{R}$;

(iii) $\left(\operatorname{supp}\left(c_{\psi_{1}\left[\Theta_{1}\right]}(q)\right)\right) \cap\left(\operatorname{supp}\left(c_{\psi_{2}\left[\Theta_{2}\right]}(q)\right)\right)=\varnothing$ and

(iv) $\left|\psi_{i}\left[\Theta_{i}\right]\right\rangle=\int_{\theta_{1}}^{\theta_{2}} C_{\psi_{i}}\left[\Theta_{i}\right](q)|q\rangle \mathrm{d} q, i=1,2$.

Then if the system is in a state $\left|\Psi^{a_{1}, a_{2}}\left[\Theta_{1}, \Theta_{2}\right]\right\rangle$ described by a wave function $\Psi^{a_{1}, a_{2}}\left(q ; \Theta_{1}, \Theta_{2}\right)=\left\langle q \mid \Psi^{a_{1}, a_{2}}\left[\Theta_{1}, \Theta_{2}\right]\right\rangle, q \in \Theta_{1} \cup \Theta_{2}$ and the value of observable $Q_{\Theta_{1}, \Theta_{2}}$ is measured once each on many identically prepared system, the average value of all the measurements will be

$$
\left\langle Q_{\Theta_{1}, \Theta_{2}}\right\rangle=\int_{\Theta_{1} \cup \Theta_{2}} q\left|\Psi^{a_{1}, a_{2}}\left(q ; \Theta_{1}, \Theta_{2}\right)\right|^{2} \mathrm{~d} q .
$$

C.V. 2. The probability of getting a result $q$ with an accuracy $\delta q$ such that $\left(q-\frac{1}{2} \delta q, q+\frac{1}{2} \delta q\right) \in \operatorname{supp}\left(c_{\psi_{1}}(q)\right) \quad$ or $\quad\left(q-\frac{1}{2} \delta q, q+\frac{1}{2} \delta q\right) \in \operatorname{supp}\left(c_{\psi_{2}}(q)\right)$ given by

$$
\int_{\left|q-q^{\prime}\right| \leq \delta q / 2}\left[\left(\left|\left\langle q^{\prime} \mid \psi_{1}^{a_{1}}\left[\Theta_{1}\right]\right\rangle\right|^{2}\right) *\left(\left|\left\langle q^{\prime} \mid \psi_{2}^{a_{2}}\left[\Theta_{2}\right]\right\rangle\right|^{2}\right)\right] \mathrm{d} q^{\prime} .
$$

Remark C.8. Note that C.IV.3 immediately follows from C.III.3. 
C.V. 3. Assume that the system is initially in the state $\left|\Psi^{a_{1}, a_{2}}\left[\Theta_{1}, \Theta_{2}\right]\right\rangle$. If on performing a measurement of $Q_{\Theta_{1}, \Theta_{2}}$ with an accuracy $\delta q$, the result is obtained in the range $\left(q-\frac{1}{2} \delta q, q+\frac{1}{2} \delta q\right)$, then the state of the system immediately after measurement given by

$$
\begin{aligned}
& \frac{\hat{P}\left(q_{i}, \delta q\right)\left|\Psi^{a_{1}, a_{2}}\left[\Theta_{1}, \Theta_{2}\right]\right\rangle}{\sqrt{\left\langle\psi\left|\hat{P}\left(q_{i}, \delta q\right)\right| \psi\right\rangle}} \\
&= \frac{\int_{\left|q-q^{\prime}\right| \leq \delta q / 2}\left(\left|q^{\prime}\right\rangle\left\langle q^{\prime} \mid \psi_{1}^{a_{1}}\left[\Theta_{1}\right]\right\rangle+\left|q^{\prime}\right\rangle\left\langle q^{\prime} \mid \psi_{2}^{a_{2}}\left[\Theta_{2}\right]\right\rangle\right) \mathrm{d} q^{\prime}}{\sqrt{\int_{\left|q-q^{\prime}\right| \leq \delta q / 2}\left[\left|\left\langle q^{\prime} \mid \psi_{1}\left[\Theta_{1}\right]\right\rangle\right|^{2}+\left|\left\langle q^{\prime} \mid \psi_{2}\left[\Theta_{2}\right]\right\rangle\right|^{2}\right] \mathrm{d} q^{\prime}}} \\
&= \frac{\int_{\left|q-q^{\prime}\right| \leq \delta q / 2}\left(a_{1}\left|q^{\prime}\right\rangle\left\langle q^{\prime} \mid \psi_{1}\left[\Theta_{1}\right]\right\rangle+a_{2}\left|q^{\prime}\right\rangle\left\langle q^{\prime} \mid \psi_{2}\left[\Theta_{2}\right]\right\rangle\right) \mathrm{d} q^{\prime}}{\sqrt{\int_{\left|q-q^{\prime}\right| \leq \delta q / 2}\left[\left|\left\langle q^{\prime} \mid \psi_{1}\left[\Theta_{1}\right]\right\rangle\right|^{2}+\left|\left\langle q^{\prime} \mid \psi_{2}\left[\Theta_{2}\right]\right\rangle\right|^{2}\right] \mathrm{d} q^{\prime}}} \in \boldsymbol{H}_{\Theta_{i}}, \\
& q_{i} \in \Theta_{i}, \quad i=1,2 .
\end{aligned}
$$

Definition C.6. Let $\boldsymbol{H}_{1,2}$ be $\boldsymbol{H}_{1,2} \triangleq \boldsymbol{H}_{\Theta_{1}} \oplus \boldsymbol{H}_{\Theta_{2}}$.

Definition C.7. Let $\left|\psi^{a}\right\rangle$ be a state $\left|\psi^{a}\right\rangle=a|\psi\rangle$, where $|\psi\rangle \in \boldsymbol{S}^{\infty},|a| \neq 1$ and $|\psi\rangle=\int_{\theta_{1}}^{\theta_{2}} c_{\psi}(q)|q\rangle$ dq. Let $\left|\psi_{a}\right\rangle$ be an state such that $\left|\psi_{a}\right\rangle \in \boldsymbol{S}^{\infty}$. States $\left|\psi^{a}\right\rangle$ and $\left|\psi_{a}\right\rangle$ is a $\hat{Q}$-equivalent: $\left|\psi^{a}\right\rangle \sim_{\hat{Q}}\left|\psi_{a}\right\rangle$ iff

$$
P\left(q, q+\mathrm{d} q ;\left|\psi^{a}\right\rangle\right)=|a|^{-2}\left|c_{\psi}\left(q|a|^{-2}\right)\right|^{2} \mathrm{~d} q=P\left(q q+\mathrm{d} q ;\left|\psi_{a}\right\rangle\right) \mathrm{d} q .
$$

C.V. For any state $\left|\psi^{a}\right\rangle=a|\psi\rangle$, where $|\psi\rangle \in \boldsymbol{S}^{\infty},|a| \neq 1$ and $|\psi\rangle=\int_{\theta_{1}}^{\theta_{2}} c_{\psi}(q)|q\rangle \mathrm{d} q$ there exist an state $\left|\psi_{a}\right\rangle \in \boldsymbol{S}^{\infty}$ such that: $\left|\psi^{a}\right\rangle \sim_{Q}\left|\psi_{a}\right\rangle$. Definition C.8. Let $\left|\psi^{a}\right\rangle$ be a state $\left|\psi^{a}\right\rangle=a|\psi\rangle$, where $|\psi\rangle \in \boldsymbol{S}^{\infty}, a \in \mathbb{C},|a| \neq 1$ and $|\psi\rangle=\int_{\theta_{1}}^{\theta_{2}} c_{\psi}(q)|q\rangle \mathrm{d} q$. Let $\left|\psi_{a}\right\rangle$ be an state such that $\left|\psi_{a}\right\rangle \in \boldsymbol{S}^{\infty}$. States $\left|\psi^{a}\right\rangle$ and $\left|\psi_{a}\right\rangle$ is a $\hat{\mathbf{Q}}$-equivalent $\left(\left|\psi^{a}\right\rangle \sim{ }_{\hat{Q}}\left|\psi_{a}\right\rangle\right)$ iff: $\left\langle\psi^{a}|\hat{Q}| \psi^{a}\right\rangle=\left\langle\psi_{a}|\hat{Q}| \psi_{a}\right\rangle$.

C.VI. For any state $\left|\psi^{a}\right\rangle=a|\psi\rangle$, where $|\psi\rangle \in \boldsymbol{S}^{\infty},|a| \neq 1$ and $|\psi\rangle=\int_{\theta_{1}}^{\theta_{2}} C_{\psi}(q)|q\rangle \mathrm{d} q$ there exists an state $\left|\psi_{a}\right\rangle \in \boldsymbol{S}^{\infty}$ such that: $\left|\psi^{a}\right\rangle{ }_{\hat{Q}}\left|\psi_{a}\right\rangle$.

\section{Appendix D. The Position Representation: Position Observable of a Particle in One Dimension}

The position representation is used in quantum mechanical problems where it is the position of the particle in space that is of primary interest. For this reason, the position representation, or the wave function, is the preferred choice of representation.

D.1. In one dimension, the position $x$ of a particle can range over the values $-\infty<x<+\infty$. Thus the Hermitean operator $\hat{x}$ corresponding to this observable will have eigenstates $|x\rangle$ and associated eigenvalues $x$ such that:

$\hat{x}|x\rangle=x|x\rangle, \quad-\infty<x<+\infty$.

D.2. As the eigenvalues cover a continuous range of values, the completeness 
relation will be expressed as an integral: $\left|\psi_{t}\right\rangle=\int_{-\infty}^{+\infty}|x\rangle\left\langle x \mid \psi_{t}\right\rangle \mathrm{d} x$, where $\left\langle x \mid \psi_{t}\right\rangle=\psi(x, t)$ is the wave function associated with the particle at each instant $t$. Since there is a continuously infinite number of basis states $|x\rangle$, these states are $\delta$-function normalized: $\left\langle x \mid x^{\prime}\right\rangle=\delta\left(x-x^{\prime}\right)$.

D.3. The operator $\hat{x}$ itself can be expressed as: $\hat{x}=\int_{-\infty}^{+\infty} x|x\rangle\langle x| \mathrm{d} x$.

Definition D.1. A connected set is a set $X \subset \mathbb{R}$ that cannot be partitioned into two nonempty subsets which are open in the relative topology induced on the set. Equivalently, it is a set which cannot be partitioned into two nonempty subsets such that each subset has no points in common with the set closure of the other.

D.4. The wave function is, of course, just the components of the state vector $\left|\psi_{t}\right\rangle \in \boldsymbol{S}^{\infty}$ with respect to the position eigenstates as basis vectors. Hence, the wave function is often referred to as being the state of the system in the position representation. The probability amplitude $\left\langle x \mid \psi_{t}\right\rangle$ is just the wave function, written $\left\langle x \mid \psi_{t}\right\rangle \triangleq \psi(x, t)$ and is such that $|\psi(x, t)|^{2} \mathrm{~d} x$ is the probability $P\left(x, t ;\left|\psi_{t}\right\rangle\right)$ of the particle being observed to have a coordinate in the range $x$ to $x+\mathrm{d} x$

Definition D.2. Let $\left|\psi_{t}^{a}\right\rangle, t \in[0,+\infty)$ be a state $\left|\psi_{t}^{a}\right\rangle=a\left|\psi_{t}\right\rangle$, where $\left|\psi_{t}\right\rangle \in \boldsymbol{S}^{\infty},|a| \neq 1$ and $\left|\psi_{t}\right\rangle=\int_{-\infty}^{+\infty} \psi(x, t)|x\rangle \mathrm{d} x$. Let $\left|\psi_{t, a}\right\rangle, t \in[0,+\infty)$ be an state such that $\left|\psi_{t, a}\right\rangle \in \boldsymbol{S}^{\infty}, t \in[0,+\infty)$. States $\left|\psi_{t}^{a}\right\rangle$ and $\left|\psi_{t, a}\right\rangle$ is $x$-equivalent $\left(\left|\psi_{t}^{a}\right\rangle \sim_{x}\left|\psi_{t, a}\right\rangle\right)$ iff

$$
P\left(x, t ;\left|\psi_{t}^{a}\right\rangle\right) \mathrm{d} x=|a|^{-2}\left|\psi\left(x|a|^{-2}, t\right)\right|^{2} \mathrm{~d} x=P\left(x, t ;\left|\psi_{t, a}\right\rangle\right) \mathrm{d} x
$$

D.5. From postulate C.5 (see Appendix C) follows: for any state $\left|\psi_{t}^{a}\right\rangle=a\left|\psi_{t}\right\rangle$, where $\left|\psi_{t}\right\rangle \in \boldsymbol{S}^{\infty},|a| \neq 1, t \in[0,+\infty)$ and $\left|\psi_{t}\right\rangle=\int_{-\infty}^{+\infty} \psi(x, t)|x\rangle \mathrm{d} x$ there exists an state $\left|\psi_{t, a}\right\rangle \in \boldsymbol{S}^{\infty}, t \in[0,+\infty)$ such that: $\left|\psi_{t}^{a}\right\rangle \sim_{x}\left|\psi_{t, a}\right\rangle$.

Definition D.2. Let $\left|\psi_{t}^{a}\right\rangle, t \in[0,+\infty)$ be a state $\left|\psi_{t}^{a}\right\rangle=a\left|\psi_{t}\right\rangle$, where $\left|\psi_{t}\right\rangle \in \boldsymbol{S}^{\infty},|a| \neq 1$ and $\left|\psi_{t}\right\rangle=\int_{-\infty}^{+\infty} \psi(x, t)|x\rangle \mathrm{d} x$. Let $\left|\psi_{t, a}\right\rangle, t \in[0,+\infty)$ be a state such that $\left|\psi_{t, a}\right\rangle \in \boldsymbol{S}^{\infty}, t \in[0,+\infty)$. States $\left|\psi_{t}^{a}\right\rangle$ and $\left|\psi_{t, a}\right\rangle$ are $\hat{x}$-equivalent $\left(\left|\psi_{t}^{a}\right\rangle \sim_{\hat{x}}\left|\psi_{t, a}\right\rangle\right)$ iff: $\left\langle\psi_{t}^{a}|\hat{x}| \psi_{t}^{a}\right\rangle=\left\langle\psi_{t, a}|\hat{x}| \psi_{t, a}\right\rangle$.

D.6. From postulate C.7 (see Appendix C) follows: for any state $\left|\psi_{t}^{a}\right\rangle=a\left|\psi_{t}\right\rangle$, where $\left|\psi_{t}\right\rangle \in \boldsymbol{S}^{\infty},|a| \neq 1, t \in[0,+\infty)$ and $\left|\psi_{t}\right\rangle=\int_{-\infty}^{+\infty} \psi(x, t)|x\rangle \mathrm{d} x$ there exists an state $\left|\psi_{t, a}\right\rangle \in \boldsymbol{S}^{\infty}, t \in[0,+\infty)$ such that: $\left|\psi_{t}^{a}\right\rangle \sim_{\hat{x}}\left|\psi_{t, a}\right\rangle$.

Definition D.3. The pure state $\left|\psi_{t}\right\rangle \in \boldsymbol{S}^{\infty}, t \in[0,+\infty),\left|\psi_{t}\right\rangle=\int_{-\infty}^{+\infty} \psi(x, t)|x\rangle \mathrm{d} x$ is a weakly Gaussian in the position representation iff

$$
|\psi(x, t)|^{2} \mathrm{~d} x=\frac{1}{\sigma_{t} \sqrt{2 \pi}} \exp \left[-\frac{\left(x-\bar{x}_{t}\right)^{2}}{\sigma_{t}^{2}}\right] \mathrm{d} x .
$$

where $\bar{x}_{t}$ and $\sigma_{t}$ are given functions which depend only on variable $t$. 
D.7. From statement D.5 it follows: for any state $\left|\psi_{t}^{a}\right\rangle=a\left|\psi_{t}\right\rangle$, where $\left|\psi_{t}\right\rangle \in \boldsymbol{S}^{\infty},|a| \neq 1, \quad t \in[0,+\infty)$ and $\left|\psi_{t}\right\rangle=\int_{-\infty}^{+\infty} \psi(x, t)|x\rangle \mathrm{d} x$ is a weakly Gaussian state there exists an weakly Gaussian state $\left|\psi_{t, a}\right\rangle \in \boldsymbol{S}^{\infty}, t \in[0,+\infty)$ such that:

$$
P\left(x, t ;\left|\psi_{t}^{a}\right\rangle\right) \mathrm{d} x=|a|^{-1}\left|\psi\left(x|a|^{-1}, t\right)\right|^{2} \mathrm{~d} x=\frac{1}{|a| \sigma_{t} \sqrt{2 \pi}} \exp \left[-\frac{\left(x-|a| \bar{x}_{t}\right)^{2}}{|a|^{2} \sigma_{t}^{2}}\right] \mathrm{d} x . \text { (D.3) }
$$

Submit or recommend next manuscript to SCIRP and we will provide best service for you:

Accepting pre-submission inquiries through Email, Facebook, LinkedIn, Twitter, etc. A wide selection of journals (inclusive of 9 subjects, more than 200 journals) Providing 24-hour high-quality service User-friendly online submission system Fair and swift peer-review system Efficient typesetting and proofreading procedure Display of the result of downloads and visits, as well as the number of cited articles Maximum dissemination of your research work

Submit your manuscript at: http://papersubmission.scirp.org/

Or contact jamp@scirp.org 